\title{
THE SLOAN DIGITAL SKY SURVEY QUASAR LENS SEARCH. II. STATISTICAL LENS SAMPLE FROM THE THIRD DATA RELEASE
}

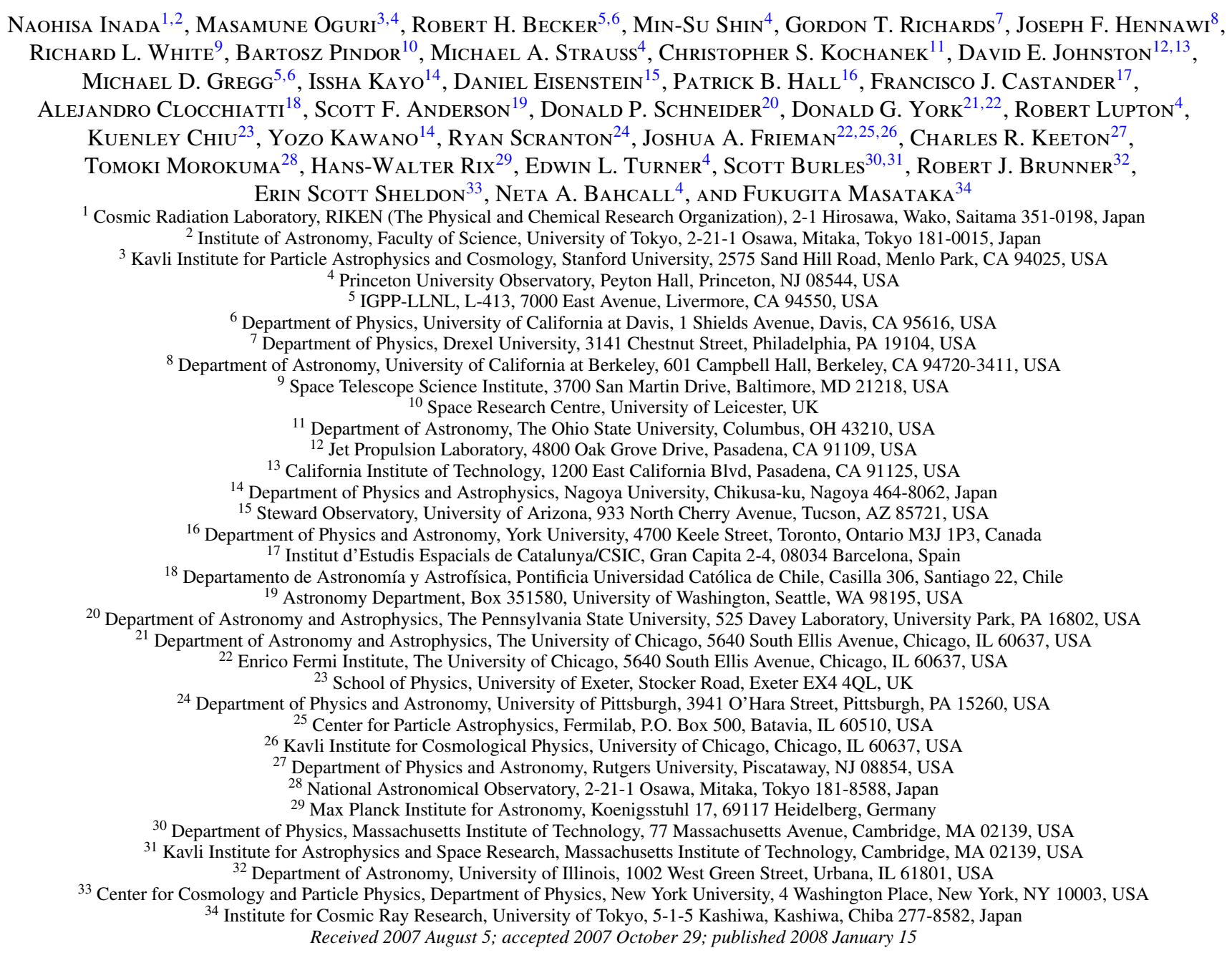

\section{ABSTRACT}

We report the first results of our systematic search for strongly lensed quasars using the spectroscopically confirmed quasars in the Sloan Digital Sky Survey (SDSS). Among 46,420 quasars from the SDSS Data Release 3 $\left(\sim 4188 \mathrm{deg}^{2}\right)$, we select a subsample of 22,683 quasars that are located at redshifts between 0.6 and 2.2 and are brighter than the Galactic extinction-corrected $i$-band magnitude of 19.1 . We identify 220 lens candidates from the quasar subsample, for which we conduct extensive and systematic follow-up observations in optical and near-infrared wavebands, in order to construct a complete lensed quasar sample at image separations between $1^{\prime \prime}$ and $20^{\prime \prime}$ and flux ratios of faint to bright lensed images larger than $10^{-0.5}$. We construct a statistical sample of 11 lensed quasars. Ten of these are galaxy-scale lenses with small image separations $\left(\sim 1^{\prime \prime}-2^{\prime \prime}\right)$ and one is a large separation $\left(15^{\prime \prime}\right)$ system which is produced by a massive cluster of galaxies, representing the first statistical sample of lensed quasars including both galaxy- and cluster-scale lenses. The Data Release 3 spectroscopic quasars contain an additional 11 lensed quasars outside the statistical sample.

Key words: cosmology: observations - gravitational lensing - quasars: general

Online-only material: machine-readable table

\section{INTRODUCTION}

Large systematic surveys of gravitationally lensed quasars are essential for various scientific applications, as shown in a recent review by Kochanek et al. (2006). For example, lensing probabilities from large homogeneous surveys, which can be estimated from the number of lenses in a statistically well-defined sample of quasars, offer a probe of cosmological parameters. 
The largest existing survey, the Cosmic-Lens All Sky Survey (CLASS; Myers et al. 2003; Browne et al. 2003), contains a total of 22 lenses discovered from high-resolution imaging of over 16,000 flat spectrum radio sources. A subset of 13 lenses from 8,958 radio sources constitutes a statistically well-defined lens sample which has been used to study cosmological parameters as well as the structure of lens galaxies (e.g., Rusin \& Tegmark 2001; Chae et al. 2002, 2006; Chen 2004; Mitchell et al. 2005). However, a drawback of radio lens surveys like CLASS is that the redshift distribution of the source population, which is a key component for statistical analyses, is poorly constrained (e.g., Muñoz et al. 2003). Thus we will benefit from complementary optical lens samples for which source populations are better understood, although the effects of absorption and emission by the lensing galaxies are larger in the optical than the radio (e.g., Falco et al. 1999). The largest existing statistical sample of optical lensed quasars is the Hubble Space Telescope (HST) snapshot survey (Maoz et al. 1993). It contains only five lenses selected from 502 bright highredshift quasars, indicating the need for much larger optical lens samples.

Larger statistical lens samples will also allow the study of the formation of structure. Quasars can be lensed by structures on scales from individual galaxies, through groups, to clusters, and therefore the image separation distribution of strongly lensed quasars from small to large separations directly reflects the hierarchical structure formation and the effects of cooling the baryons (e.g., Kochanek \& White 2001; Oguri 2006). Unfortunately, the probability of quasars strongly lensed by clusters is 1-2 orders of magnitudes smaller than that by galaxies, so we need large homogeneous surveys to study the full image separation distribution. Indeed, despite searching for them explicitly, no cluster-scale lens was discovered in the CLASS (Phillips et al. 2001).

The main purpose of the Sloan Digital Sky Survey Quasar Lens Search (SQLS; Oguri et al. 2006, hereafter Paper I) is to construct a large sample of lensed quasars in the optical. It is made possible by the large spectroscopic quasar catalog obtained from the data of the Sloan Digital Sky Survey (SDSS; York et al. 2000). Lens candidates are selected morphologically among the spectroscopically confirmed SDSS quasars. Additional lens candidates are selected by looking for companion objects to the SDSS quasars that have similar colors. Our selection algorithms have been tested against simulated SDSS images; this allows accurate quantification of the selection function (see Paper I). A number of previous strong lens discoveries (Inada et al. 2007a, and references therein) indicate the effectiveness of our candidate-selection algorithm.

In this paper, we present the statistical sample of strongly lensed quasars, constructed from the SDSS Data Release 3 (DR3; Abazajian et al. 2005) spectroscopic quasar catalog (Schneider et al. 2005). Lens candidates are selected according to the algorithms presented in Paper I. We conduct extensive follow-up observations for these candidates with various facilities in order to test the hypothesis that they are lensed, and to make a complete lens sample. Cosmological constraints from this statistical sample will be reported in Paper III of this series (Oguri et al. 2008a).

The structure of this paper is as follows. We describe the construction of the source quasar sample in Section 2, and the selection of lens candidates in Section 3. Results of our followup observations for the candidates are summarized in Section 4. Section 5 lists lensed quasars in the statistical quasar subsample as well as those included in the DR3 quasar catalog. Finally we summarize our results in Section 6.

\section{THE SOURCE QUASAR SAMPLE}

The SDSS is a combination of photometric and spectroscopic surveys of a quarter of the entire sky (York et al. 2000). It uses a dedicated wide-field ( $3^{\circ}$ field of view) $2.5 \mathrm{~m}$ telescope (Gunn et al. 2006) at the Apache Point Observatory in New Mexico, USA. Photometric observations (Gunn et al. 1998; Tucker et al. 2006) consist of imaging in five broadband filters (Fukugita et al. 1996). The data are processed and analyzed automatically by the photometric pipeline (Lupton et al. 2001; Lupton 2005). Targets for spectroscopy are selected according to selection algorithms applied to the imaging data (see Richards et al. (2002) for the quasar target selection algorithm). Spectra of these targets are obtained with a multi-fiber spectrograph (wavelength range between $3800 \AA$ and $9200 \AA$ at a resolution of $R \sim 1,800$ ). The astrometric accuracy of the imaging data is better than about $0.1 \mathrm{rms}$ per coordinate (Pier et al. 2003) and photometric zeropoint errors are less than about 0.03 magnitude over the entire survey area (Hogg et al. 2001; Smith et al. 2002; Ivezić et al. 2004; Padmanabhan et al. 2008). The data release papers (Stoughton et al. 2002; Abazajian et al. 2003, 2004, 2005; Adelman-McCarthy et al. 2006, 2007) describe the contents of the SDSS data releases.

We start with a sample of 46,420 spectroscopically confirmed quasars in the SDSS DR3 quasar catalog (Schneider et al. 2005). The area covered by the spectroscopy is $4188 \mathrm{deg}^{2}$. The sample contains quasars from $z=0.08$ to 5.41 with a median redshift of 1.47. This is not a well-defined quasar sample for lens surveys, as it includes objects selected with a wide variety of techniques. For example, when high-redshift quasar candidates $(z>2.2)$ are targeted for SDSS spectroscopy, they are required to be point sources, leading to a strong bias against selecting smallseparation lenses. We focus on the low redshift $(z<2.2)$ and bright $\left(i_{\text {cor }}<19.1\right.$; here $i_{\text {cor }}$ is the point-spread function (PSF) magnitude corrected for Galactic extinction from the maps of Schlegel et al. (1998)) quasars of the main quasar sample, which are known to have high completeness regardless of whether they are resolved or unresolved (Vanden Berk et al. 2005; Richards et al. 2006). Thus the quasars with $z<2.2$ and $i_{\text {cor }}<19.1$ should have no explicit biases against gravitational lenses. We further restrict the redshift range to $0.6<z<2.2$ to eliminate lower redshift, intrinsically extended quasars, and exclude quasars with SDSS images of poor seeing (PSF_WIDTH > $\left.1^{\prime \prime} .8\right)$ in which the identification of close lens pairs is difficult. Paper I discusses the selection of the source quasars in greater detail. These criteria produce a subsample of 22,683 quasars ${ }^{35}$ suitable for lens statistics. From this quasar subsample, we construct a statistical sample of lensed quasars.

\section{LENS CANDIDATE SELECTION}

We illustrate the SQLS candidate selection procedure in Figure 1. As discussed in Paper I, we use two different selection methods (morphological and color selection), in order to identify both galaxy- and cluster-scale lens candidates. For candidates selected by each approach, we apply several additional selection criteria to construct a final lens candidate sample appropriate for detailed follow-up on other facilities. We explain

\footnotetext{
35 This includes one quasar (SDSS J094222.89+102025.3), which we missed in Paper I.
} 


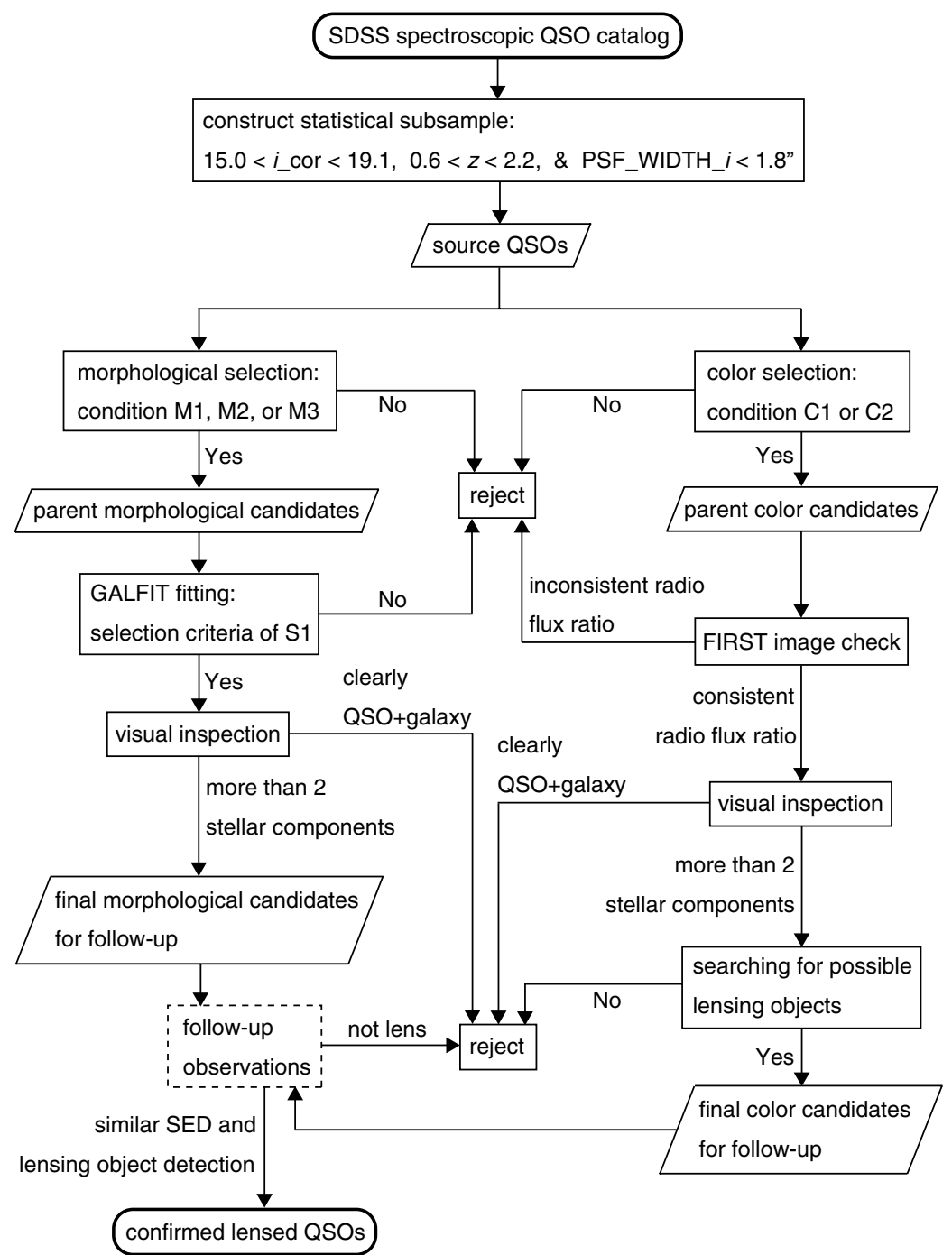

Figure 1. Flowchart of the candidate selection procedure of the SQLS. First we construct a statistical subsample of quasars (source QSOs) from the SDSS spectroscopic quasar catalog. The specific selection criteria (M1-M3, C1-C2, and S1) are given in Paper I. The details of the additional selection criteria are described in Section 3. Table 1 presents the numbers of the source quasars, parent candidates, objects rejected at each step, and final follow-up candidates.

these additional criteria for morphological candidates in Section 3.1 and those for color candidates in Section 3.2. These two selection algorithms are not exclusive with each other, since the (deblended) quasar component of a color candidate could be a morphological candidate. The numbers of candidates selected/removed by each approach are summarized in Table 1 . We finally identify 220 lensed quasar candidates for follow-up from the 22,683 source quasars.

\subsection{Morphological Selection}

The morphological selection algorithm is intended to discover galaxy-scale $(\theta \lesssim 2$ ". 5$)$ lensed quasars that the SDSS photometric pipeline did not deblend into multiple components. In Paper I, we showed that such lens candidates can be identified by searching for quasars that are not well fit by the PSF in each SDSS field. Thus we select the galaxy-scale lens candidates based on the goodness of fit of a quasar image to the PSF model in each field (star_L). Different criteria of star_L are used according to the object classification (objc_type), which is set by the difference between the PSF and model magnitudes in the SDSS data. The specific criteria (M1, M2, or M3) are given in Paper
Table 1

Numbers of Candidates

\begin{tabular}{lr}
\hline \hline & Number \\
\hline Source quasars & 22,683 \\
Parent morphological candidates & 649 \\
Rejected by GALFIT fitting & -552 \\
Rejected by visual inspection & -7 \\
Final morphological candidates for follow-up & 90 \\
Parent color candidates & 227 \\
Rejected by FIRST image check & -16 \\
Rejected by visual inspection & -16 \\
Rejected by searching for possible lensing objects & -63 \\
Final color candidates for follow-up & 132 \\
Final total candidates for follow-up & 220 \\
\hline
\end{tabular}

Note. Two candidates are selected by both the morphological and color selection algorithms.

I. These "morphological selection" criteria identify 649 quasars ( $\sim 3 \%$ of the source quasars) as lens candidates. 
As discussed in Paper I, a significant fraction of the candidates selected by the algorithm are single quasars. In addition, the candidates contain superpositions of quasars with foreground galaxies or stars. Therefore, we use two additional criteria described below in order to exclude most of these false positives before performing any subsequent observations. In the first criterion, we fit the SDSS $u$ - and $i$-band images of each candidate with two PSFs using the GALFIT software (Peng et al. 2002). When a single quasar is fitted with two PSFs, the result tends to be unusual in the sense that the two fitted PSFs have a very small separation $\left(\ll 1^{\prime \prime}\right)$ and similar magnitudes, or a moderate separation $\left(\gtrsim 1^{\prime \prime}\right)$ and very different magnitudes. In addition, when a single quasar plus star/galaxy system is fitted with two PSFs, the results from the SDSS $u$ - and $i$-band images tend to be very different because a quasar at $0.6<z<2.2$ is usually much bluer than either a star or a galaxy. In particular, such quasar plus star/galaxy systems may result in very large $u$-band flux ratios since the galaxy/star component tends to be very faint in the $u$-band. Thus we can eliminate a significant fraction of these false positives by making cuts to the fitted separations and flux ratios (see Paper I for selection criterion S1). This procedure ("GALFIT fitting") successfully removes $\sim 85 \%$ of the candidates. Most of the rejections are single isolated quasars but, as expected, they also include several quasar-star and quasar-galaxy pairs.

For the second criterion, we inspect the SDSS images of the remaining candidates and eliminate those which appear to be chance superpositions of a quasar and a galaxy ("visual inspection"). This visual inspection eliminates about $10 \%$ of the candidates left after the GALFIT modeling and yields 90 morphological (galaxy-scale) candidates that require further investigation.

\subsection{Color Selection}

Larger separation $\left(\theta \gtrsim 2^{\prime \prime} .5\right)$ lenses created by groups or clusters of galaxies are accurately deblended by the SDSS photometric pipeline, and therefore can be selected by comparing the colors of each quasar to those of nearby objects ("color selection"). The idea is similar to the Hennawi et al. (2006) approach for finding binary quasars in the SDSS, but we modify the color selection criteria to allow for differential extinction between lensed images. These criteria are discussed in detail in Paper I, but we have slightly broadened the limits to include image separations of $\theta<20$." 1 and $i$-band magnitude differences of $\Delta i<1.3$, considering the typical uncertainties in the quasar positions $\left(0 .{ }^{\prime \prime} 1\right)$ and magnitudes $(0.05)$ in the SDSS data. We identified 227 quasar pairs based on the criteria.

We first test the lensing hypothesis for these candidates by comparing the radio flux ratios from the Faint Images of the Radio Sky at Twenty centimeters survey (FIRST; Becker et al. 1995) with the optical flux ratios ("FIRST image check"). We eliminate $10 \%$ of the candidates with separations larger than $6^{\prime \prime}$ (set by the $\sim 5^{\prime \prime}$ resolution of the FIRST) in which one component is radio-loud with a flux well above the FIRST survey limits and the other is not. Such pairs are either binary quasars or quasar-star pairs (Kochanek et al. 1999).

Next we check the SDSS images of the candidates ("visual inspection"). Since we select both point sources and extended sources as the nearby objects in the color selection stage, quasar plus galaxy systems are sometimes selected as candidates. The purpose of visual inspection in the color selection algorithm is to eliminate the candidates whose companion objects to the SDSS

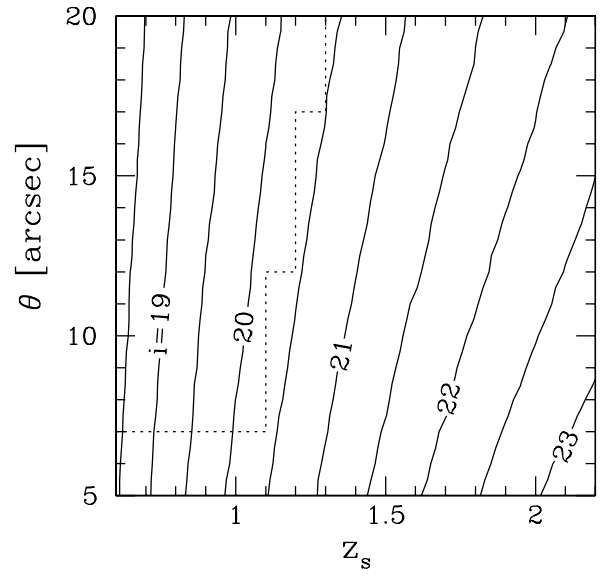

Figure 2. $i$-band magnitude limit of the lensing objects (defined such that $99 \%$ of simulated lenses are caused by galaxies brighter than the limit) in the $z_{s}-\theta$ plane, where $z_{s}$ denotes the source (quasar) redshift. The limit is computed using the halo model of Oguri (2006). The dotted line indicates the limits of the source redshift and image separation (Equation (1)): for candidates which lie in the region of Equation (1), we search for possible lensing galaxies in the SDSS $i$-band images and reject the candidates if no galaxy is seen among the components. See text for more details.

quasars clearly appear to be galaxies. At this step an additional $\sim 10 \%$ of the candidates are excluded.

In addition, for low-redshift candidates we can search for possible lensing galaxies in the SDSS images ("searching for possible lensing objects"). To determine the criteria, we compute the expected magnitudes of the lensing objects using the halo model of Oguri (2006). Specifically, we define the magnitude limit of the lensing galaxy (or the central galaxy of the lensing cluster for a cluster-scale lens candidate) such that $99 \%$ of simulations have a lensing galaxy brighter than that magnitude limit, and compute the magnitude limit as a function of the image separation and source (quasar) redshift. In the model, halos are linked to galaxies by adopting a universal scaling relation (with scatter) between masses of halos and luminosities of galaxies. We ignore the redshift evolution of the mass-luminosity relation, which provides conservative estimates of the minimum luminosity since standard passive evolution predicts that galaxies were brighter in the past. The luminosity is converted to observed magnitude using the $K$ correction for elliptical galaxies in Fukugita et al. (1995). Figure 2 shows the magnitude limit as a function of image separations and quasar redshifts. We check for a lensing galaxy in the SDSS image when its expected magnitude is brighter than $i=20.5$ (corresponding to $\sim 0.5 L_{*}$ for a typical lens redshift); given the magnitude limit of the SDSS images, $i_{\text {lim }} \sim 21.5$, the choice should be very conservative. In practice, we examine candidates with low redshifts and large separations defined by the region shown in Figure 2:

$$
\begin{array}{ll}
z_{s}<1.1 & \text { for } \quad 7^{\prime \prime}<\theta<12^{\prime \prime}, \\
z_{s}<1.2 & \text { for } 12^{\prime \prime}<\theta<17^{\prime \prime}, \\
z_{s}<1.3 & \text { for } 17^{\prime \prime}<\theta,
\end{array}
$$

where $z_{s}$ is the redshift of the source quasar. The candidate which lies in the region and lacks a possible lensing object in the SDSS image is rejected. Roughly $30 \%$ of the candidates fail this test, leaving 132 color (larger-separation) candidates. These 132 objects constitute the final color candidates for additional investigation. Among these, two candidates were also selected by the morphological algorithm, which is consistent with our 
simulation that predicts $\sim 10 \%$ of objects with image separations of $1^{\prime \prime} .5 \lesssim \theta \lesssim 3^{\prime \prime} .0$ are selected by both algorithms (see Paper I). Thus the total number of candidates for follow-up is 220 .

\section{FOLLOW-UP OBSERVATIONS}

The final morphological and color candidates and the summary of their observations are shown in Tables 2 and 3, respectively. In this section, we describe the follow-up observations and how we decide on the lens nature of each candidate. We also note several interesting objects discovered in the course of our lens search.

\subsection{Basic Strategy}

Before conducting any subsequent observations, we first check if the candidates have been studied before with the NASA/IPAC Extragalactic Database (NED). Three of the 220 candidates are previously known gravitational lens systems for which no follow-up observations were necessary. One system, SDSS J133945.37+000946.1, turns out to be a quasar pair at different redshifts (Croom et al. 2004).

The rest of the candidates are investigated to test their lensing hypotheses. These observations consist of optical spectroscopy, optical imaging, and near-infrared imaging, conducted at the following facilities: the University of Hawaii $2.2 \mathrm{~m}$ telescope (UH88), the Astrophysical Research Consortium $3.5 \mathrm{~m}$ telescope (ARC $3.5 \mathrm{~m}$ ), the Keck I and II telescopes, the United Kingdom Infra-Red Telescope (UKIRT), the Subaru telescope, the Magellan Consortium's Walter Baade $6.5 \mathrm{~m}$ telescope (WB $6.5 \mathrm{~m}$ ), the HST, the MDM $2.4 \mathrm{~m}$ telescope (MDM $2.4 \mathrm{~m}$ ), the MMT Observatory, the European Southern Observatory $3.6 \mathrm{~m}$ telescope (ESO $3.6 \mathrm{~m}$ ), the New Technology Telescope (NTT), and the WIYN telescope.

The SDSS images have moderate observing conditions (typically $\sim 1$ ".3 seeing) and a short exposure time (about $55 \mathrm{~s}$ ), making it difficult in many cases to determine whether a lensing galaxy is present. Therefore, for small-separation lens candidates we usually start by acquiring deeper optical $(i$ or $I$ ) or infrared $(H$ or $K)$ images under good seeing conditions $\left(\sim 0^{\prime \prime} .5\right.$ $\left.1^{\prime \prime} 0\right)$. Some of the candidates turn out to be single quasars or quasar-galaxy pairs, and therefore they are rejected rather easily. If the candidates consist of two (or more) stellar components, we take optical and/or infrared images that are deep enough to locate lensing galaxies. The typical magnitude limits for extended objects are $I \sim 23.0, H \sim 18.5$, and $K \sim 20.0$. Additional images with bluer filters may be taken in order to better separate multiple components. Candidates that do not exhibit any residuals after subtracting stellar components are rejected based on the absence of the lensing object. Some of our candidates are rejected simply by the fact that the separation of the two stellar components is smaller than $1^{\prime \prime}$, since we set the minimum image separation of our complete lens sample to be $1^{\prime \prime}$ (i.e., some of the "rejected" candidates with $\theta<1$ " could in fact be gravitational lenses; see Section 4.2). If the data reveal stellar components with similar colors and a possible lensing galaxy, we try to obtain spectra of the multiple stellar components to determine whether their spectral energy distributions (SEDs) are similar. We detect possible lensing objects only for 9 candidates out of 81 morphological candidates with deep images. Seven of them turn out to be new lenses from the SDSS (see Section 5 and Table 2), and the other two are confirmed to be binary quasars (SDSS J084710.40-001302.6 and SDSS J100859.55+035104.4; see Section 4.2). We note that in ad- dition to the candidates with possible lensing objects we conduct spectroscopic observations for any candidates which have stellar components with very similar colors in imaging data (including the SDSS images) even if they are rejected based on the absence of lensing objects. We perform this spectroscopic observation in order to test the validity of the rejection criterion. This includes SDSS J093207.15+072251.3 (see Section 4.2), and SDSS J112012.11+671116.0 (Pindor et al. 2006) that are confirmed to be binary quasar pairs with indistinguishable redshifts.

Our strategy for follow-up of large-separation lens candidates is similar to the above process. We either acquire spectra of the two components to check their SEDs or deep optical/infrared images to search for any possible lensing galaxies or clusters. Rejection based upon spectroscopic observations is straightforward when the candidates are quasar-star pairs or quasar-quasar pairs at different redshifts. Some of the candidates turn out to be binary quasars, as reported in Hennawi et al. (2006). In some cases, candidates turn out to be quasar pairs at quite similar redshifts. For these sources, we use deep optical/infrared follow-up images to search for any signature of a lensing galaxy or a lensing cluster. As well as the small-separation lens candidates, we perform spectroscopic observations regardless of the existences of the lensing objects particularly when the colors of the two components are very similar. Most of them are quasar-quasar pairs at different redshifts. Two of them, SDSS J090955.54+580143.2 and SDSS J211102.60+105038.3, turn out to be quasar pairs with indistinguishable redshifts, but are rejected based on the differences of the SEDs (see Section 4.2).

To summarize, we identify a candidate as a lens system when the following three conditions are met: (i) the stellar components have the same redshifts within the measurement uncertainty; (ii) their SEDs are reasonably similar; (iii) a galaxy or a cluster/group of galaxies is detected between the stellar components. When candidates have four stellar images, we do not always require conditions (i) and (ii), because the object's lensing nature is obvious from its characteristic image configuration. The fact that we use the existence of the lensing object for the judgment suggests that our selection may be against hypothetical dark lenses (e.g., Rusin 2002) that have anomalously high mass-to-light ratios. However, since our candidates with very similar colors tend to be rejected spectroscopically (see above and Section 4.2) rather than by the absence of lensing objects alone, we believe that our followup strategy is reasonably effective in locating such dark lenses as well.

\subsection{Notes on Individual Objects}

Below we note several interesting candidates which have not been discussed elsewhere in the literature. In addition to the objects discussed below, at least 30 objects out of our 220 candidates are confirmed to be pairs of quasars; 9 out of the 30 pairs have already been reported in Hennawi et al. (2006). Furthermore, there are nine candidates whose image separations turned out to be $\theta<1^{\prime \prime}$ in the follow-up studies. They include the known lensed quasar FBQ1633+3134 (Morgan et al. 2001). These systems are not included in the statistical lens sample and therefore we did not perform any further spectroscopy or deeper imaging. Although our current follow-up images do not show any possible lensing objects for any of the candidates, lensing galaxies of subarcsecond lenses are expected and observed to be faint. Very deep and high-resolution images are necessary 
Table 2

Morphological Candidates

\begin{tabular}{|c|c|c|c|c|c|c|c|c|}
\hline Object & $z^{\mathrm{a}}$ & $i_{\text {cor }}^{\mathrm{b}}$ & $\theta_{\text {SDSS }^{c}}$ & $\Delta i^{\mathrm{c}}$ & Image $^{\mathrm{d}}$ & Spec $^{\mathrm{d}}$ & Comment & Ref. \\
\hline SDSS J001125.75-105710.4 & 0.641 & 18.95 & 0.48 & 0.70 & $\mathrm{UF}(K)$ & $\cdots$ & Single QSO & $\cdots$ \\
\hline SDSS J004242.00+135450.0 & 1.622 & 16.72 & 0.56 & 0.81 & $\mathrm{UF}(K)$ & $\cdots$ & Single QSO & $\ldots$ \\
\hline SDSS J011229.41+151213.9 & 1.957 & 18.86 & 1.12 & 0.30 & $\mathrm{WF}(i)$ & $\cdots$ & QSO+galaxy & $\cdots$ \\
\hline SDSS J012259.49+151147.2 & 1.276 & 18.17 & 0.45 & 0.36 & $\mathrm{UF}(K)$ & $\cdots$ & Single QSO & $\ldots$ \\
\hline SDSS J020707.59-100541.6 & 0.662 & 18.78 & 0.48 & 0.10 & $\mathrm{UF}(K)$ & $\cdots$ & Single QSO & $\cdots$ \\
\hline SDSS J021249.59+003448.7 & 1.222 & 18.86 & 1.69 & 0.73 & $8 \mathrm{k}(I), \mathrm{WF}(i)$ & WF & QSO+galaxy & $\cdots$ \\
\hline SDSS J021645.80-010204.8 & 1.087 & 18.55 & 0.53 & 1.35 & $\mathrm{UF}(K)$ & $\cdots$ & Single QSO & $\cdots$ \\
\hline SDSS J024634.09-082536.1 & 1.685 & 17.77 & 1.03 & 1.10 & $\operatorname{AC}(V, I), \operatorname{Ma}(i), \operatorname{NR}\left(K^{\prime}\right), \operatorname{NM}(H)$ & ES & SDSS Lens & 1 \\
\hline SDSS J034601.93-070024.2 & 0.630 & 19.09 & 0.74 & 1.37 & $\mathrm{UF}(K)$ & $\ldots$ & Extended single QSO & $\ldots$ \\
\hline SDSS J034801.20-070416.9 & 1.959 & 18.10 & 1.54 & 1.54 & $\mathrm{UF}(K)$ & $\ldots$ & No lensing object & $\ldots$ \\
\hline SDSS J041254.86-062049.8 & 1.266 & 18.96 & 0.48 & 0.58 & $\mathrm{UF}(K)$ & $\ldots$ & Single QSO & $\ldots$ \\
\hline SDSS J072843.03+370834.9 & 1.404 & 18.97 & 1.34 & 0.08 & $\mathrm{WF}(i)$ & & No lensing object & \\
\hline SDSS J073406.75+273355.6 & 1.916 & 16.87 & 2.48 & 0.27 & $\ldots$ & DA & QSO+star & $\cdots$ \\
\hline SDSS J074352.62+245743.6 & 2.166 & 19.01 & 1.02 & 1.14 & $\mathrm{WF}(i)$ & $\cdots$ & No lensing object & $\cdots$ \\
\hline SDSS J080002.76+405927.1 & 1.624 & 19.00 & 0.80 & 1.27 & $8 \mathrm{k}(V)$ & $\cdots$ & Single QSO & $\ldots$ \\
\hline SDSS J083530.89+054240.7 & 1.696 & 18.50 & 0.99 & 0.18 & $\operatorname{RE}(r), \operatorname{WF}(i), \mathrm{QU}(H)$ & $\cdots$ & No lensing object & $\ldots$ \\
\hline SDSS J083956.19+410950.9 & 0.629 & 19.08 & 0.73 & 1.96 & $\mathrm{WF}(i)$ & $\ldots$ & Single QSO & $\ldots$ \\
\hline SDSS J084512.74+543421.5 & 1.285 & 18.53 & 1.50 & 0.82 & $\ldots$ & MS & QSO+star & 2 \\
\hline SDSS J084710.40-001302.6 & 0.627 & 18.60 & 0.95 & 0.43 & $\operatorname{Te}(I)$ & LR & QSO pair $(z=0.626,0.627)$ & $\cdots$ \\
\hline SDSS J084856.08+011540.0 & 0.646 & 18.86 & 1.37 & 0.53 & $\operatorname{Ma}(i)$ & ES & QSO+galaxy+star & $\cdots$ \\
\hline SDSS J085122.37+472249.0 & 0.894 & 18.80 & 1.47 & 0.03 & $\mathrm{WF}(i)$ & $\cdots$ & No lensing object & $\cdots$ \\
\hline SDSS J085643.71+413444.8 & 0.756 & 17.75 & 0.55 & 0.59 & $8 \mathrm{k}(V)$ & $\cdots$ & Single QSO & $\cdots$ \\
\hline SDSS J091301.03+525928.9 & 1.377 & 16.17 & 1.12 & 0.42 & $\ldots$ & $\ldots$ & Known lens (SBS 0909) & 3 \\
\hline SDSS J092455.79+021924.9 & 1.523 & 18.12 & 1.34 & 0.64 & $\operatorname{Ma}(u, g, r, i)$ & ES & SDSS lens & 4 \\
\hline SDSS J092528.68+071442.7 & 1.630 & 18.13 & 0.55 & 0.68 & $8 \mathrm{k}(V)$ & $\ldots$ & Single QSO & $\ldots$ \\
\hline SDSS J093207.15+072251.3 & 1.993 & 18.96 & 1.42 & 1.95 & $\operatorname{RE}(r), 8 \mathrm{k}(I), \mathrm{UF}(K), \mathrm{QU}(H)$ & ES & QSO pair $(z=1.994,1.994)$ & $\ldots$ \\
\hline SDSS J094945.68+632622.9 & 0.650 & 17.72 & 0.41 & 1.26 & $\mathrm{Te}(I)$ & $\cdots$ & Single QSO & $\cdots$ \\
\hline SDSS J095324.39+570319.5 & 0.619 & 18.81 & 2.57 & 0.14 & $8 \mathrm{k}(V), \mathrm{Te}(I)$ & $\cdots$ & No lensing object & $\cdots$ \\
\hline SDSS J100229.46+444942.7 & 2.052 & 18.39 & 0.72 & 0.25 & $\operatorname{RE}(r), \operatorname{Te}(I), \operatorname{NR}\left(K^{\prime}\right), \mathrm{QU}(H)$ & $\cdots$ & $\theta=0 ! ' 8$ & $\cdots$ \\
\hline SDSS J100327.37+595804.0 & 1.138 & 17.71 & 1.19 & 0.09 & $\mathrm{FO}(V, I)$ & $\cdots$ & No multiple point sources & $\cdots$ \\
\hline SDSS J100859.55+035104.4 & 1.746 & 19.09 & 0.98 & 1.18 & $\operatorname{RE}(r), \operatorname{Te}(I), \mathrm{UF}(K), \mathrm{QU}(H)$ & FO & QSO pair $(z=1.745,1.740)$ & $\cdots$ \\
\hline SDSS J102111.02+491330.3 & 1.720 & 18.97 & 1.03 & 0.55 & $8 \mathrm{k}(I), \mathrm{NR}\left(K^{\prime}\right)$ & MS & SDSS Lens & 2 \\
\hline SDSS J103244.09+084022.5 & 0.604 & 19.05 & 0.87 & 2.05 & $\mathrm{SC}(V, R)$ & $\cdots$ & Single & $\cdots$ \\
\hline SDSS J104716.39+055159.5 & 0.891 & 18.51 & 0.54 & 1.50 & $\mathrm{Op}(I)$ & $\cdots$ & $\theta=0 ! 4$ & $\cdots$ \\
\hline SDSS J105337.63+074257.0 & 0.635 & 18.96 & 2.19 & 1.08 & $\mathrm{Op}(I)$ & $\cdots$ & Single QSO & $\cdots$ \\
\hline SDSS J111348.65+494522.4 & 0.659 & 19.03 & 0.68 & 0.14 & $\mathrm{Op}(I)$ & $\cdots$ & Extended single QSO & $\cdots$ \\
\hline SDSS J111557.64+040224.3 & 0.668 & 18.23 & 0.61 & 1.79 & $\mathrm{Op}(I)$ & $\cdots$ & Single QSO & $\cdots$ \\
\hline SDSS J112012.11+671116.0 & 1.494 & 18.46 & 1.71 & 1.40 & $8 \mathrm{k}(I), \operatorname{AR}(H)$ & MS & QSO pair $(z=1.494,1.494)$ & 2,5 \\
\hline SDSS J112241.58+641601.2 & 1.433 & 18.31 & 1.40 & 0.19 & $8 \mathrm{k}(V), \mathrm{Op}(I)$ & $\cdots$ & No lensing object & $\cdots$ \\
\hline SDSS J113236.06+030335.1 & 1.766 & 18.42 & 3.81 & 1.18 & $\mathrm{QU}(H)$ & $\ldots$ & No lensing object & $\ldots$ \\
\hline SDSS J113352.61+035300.5 & 1.780 & 16.90 & 0.41 & 0.92 & $\mathrm{Op}(I)$ & $\cdots$ & Single QSO & $\cdots$ \\
\hline SDSS J113613.37+033840.9 & 1.188 & 18.50 & 1.65 & 0.24 & $\cdots$ & MS & QSO+star & 2 \\
\hline SDSS J115244.06+571202.1 & 1.603 & 17.92 & 0.41 & 1.24 & $8 \mathrm{k}(V)$ & $\cdots$ & Single QSO & $\cdots$ \\
\hline SDSS J121244.33+091208.1 & 1.686 & 18.63 & 2.04 & 1.37 & $\mathrm{QU}(H)$ & $\mathrm{EF}$ & QSO pair $(z=1.686,1.600)$ & $\cdots$ \\
\hline SDSS J122558.45-005226.2 & 0.963 & 18.84 & 0.46 & 2.04 & $8 \mathrm{k}(V)$ & $\cdots$ & Single QSO & $\cdots$ \\
\hline SDSS J122608.02-000602.2 & 1.125 & 18.23 & 1.21 & 0.80 & $\operatorname{Ma}(g, i), \operatorname{AC}(V, I), \operatorname{NM}(H)$ & DW & SDSS Lens & 6 \\
\hline SDSS J123020.41+452047.6 & 2.112 & 18.20 & 0.55 & 1.42 & $8 \mathrm{k}(V)$ & $\ldots$ & Single QSO & $\ldots$ \\
\hline SDSS J123844.79+105622.2 & 1.305 & 18.54 & 0.97 & 0.92 & $\mathrm{Op}(I)$ & $\cdots$ & QSO+galaxy & $\cdots$ \\
\hline SDSS J123846.69+644836.5 & 1.558 & 17.66 & 1.30 & 1.09 & $8 \mathrm{k}(V), \mathrm{Te}(I)$ & $\cdots$ & QSO+galaxy & $\cdots$ \\
\hline SDSS J124803.09+611628.1 & 1.585 & 18.76 & 0.63 & 1.57 & $8 \mathrm{k}(V)$ & $\cdots$ & Single QSO & $\cdots$ \\
\hline SDSS J125141.90+031140.9 & 1.223 & 18.69 & 1.73 & 1.02 & $\cdots$ & MS & QSO+star & 2 \\
\hline SDSS J125617.97+584550.0 & 1.205 & 17.88 & 1.19 & 0.83 & $8 \mathrm{k}(V), \mathrm{Op}(I)$ & $\cdots$ & No lensing object & $\cdots$ \\
\hline SDSS J125758.84+623834.2 & 2.136 & 18.50 & 0.72 & 1.47 & $8 \mathrm{k}(V)$ & $\cdots$ & $\theta=0 . \prime 4$ & $\cdots$ \\
\hline SDSS J130337.87+505824.5 & 2.104 & 18.68 & 1.89 & 1.39 & $8 \mathrm{k}(I)$ & $\cdots$ & QSO+galaxy & $\ldots$ \\
\hline SDSS J131815.12+012450.6 & 0.688 & 18.04 & 0.41 & 1.15 & $8 \mathrm{k}(V)$ & $\cdots$ & Single QSO & $\cdots$ \\
\hline SDSS J133222.62+034739.9 & 1.438 & 17.89 & 0.99 & 0.82 & $\mathrm{SC}(i), \mathrm{QU}(H)$ & FO & SDSS Lens & 7 \\
\hline SDSS J133512.10+052732.4 & 1.958 & 18.98 & 0.79 & 0.32 & $8 \mathrm{k}(V), \mathrm{Op}(I)$ & $\cdots$ & $\theta=0 ! .8$ & $\cdots$ \\
\hline SDSS J133534.79+011805.5 & 1.571 & 17.53 & 1.59 & 1.16 & $\mathrm{SC}(i), \mathrm{NR}\left(K^{\prime}\right)$ & EM & SDSS Lens & 8 \\
\hline SDSS J133632.95+631425.0 & 1.799 & 17.21 & 0.58 & 1.98 & $8 \mathrm{k}(I)$ & $\cdots$ & Single QSO & $\cdots$ \\
\hline SDSS J140159.74+414156.2 & 1.702 & 17.91 & 1.43 & 2.19 & $\mathrm{QU}(H)$ & $\cdots$ & QSO+galaxy+galaxy & $\cdots$ \\
\hline SDSS J140622.44-011230.7 & 1.154 & 18.84 & 1.15 & 1.18 & $8 \mathrm{k}(V), \mathrm{Op}(I)$ & $\cdots$ & No lensing object & $\cdots$ \\
\hline SDSS J141647.60+630251.4 & 2.034 & 18.97 & 0.70 & 1.10 & $\mathrm{Op}(I)$ & $\cdots$ & Single QSO & $\cdots$ \\
\hline SDSS J142011.60+003711.9 & 0.727 & 18.10 & 0.46 & 0.83 & $8 \mathrm{k}(I)$ & $\cdots$ & Single QSO & \\
\hline SDSS J142931.58+012123.5 & 1.518 & 18.99 & 2.13 & 1.67 & $\mathrm{SC}(R)$ & $\cdots$ & QSO+galaxy & \\
\hline
\end{tabular}


Table 2

(Continued)

\begin{tabular}{|c|c|c|c|c|c|c|c|c|}
\hline Object & $z^{\mathrm{a}}$ & $i_{\text {cor }}{ }^{b}$ & $\theta_{\text {SDSS }}{ }^{\mathrm{c}}$ & $\Delta i^{\mathrm{c}}$ & Image $^{\mathrm{d}}$ & Spec $^{\mathrm{d}}$ & Comment & Ref. \\
\hline SDSS J143239.09+510431.6 & 1.193 & 18.94 & 0.68 & 1.59 & $8 \mathrm{k}(V), \mathrm{Op}(I)$ & $\cdots$ & $\theta=0 . .6$ & $\cdots$ \\
\hline SDSS J143826.72+642859.8 & 1.218 & 18.08 & 1.19 & 1.01 & $8 \mathrm{k}(I)$ & $\ldots$ & QSO+galaxy & $\ldots$ \\
\hline SDSS J144135.56+511023.1 & 1.556 & 18.98 & 1.44 & 1.39 & $8 \mathrm{k}(I)$ & $\cdots$ & QSO+galaxy & $\cdots$ \\
\hline SDSS J144654.77+610248.7 & 1.760 & 17.72 & 0.50 & 0.92 & $8 \mathrm{k}(V)$ & $\ldots$ & Single QSO & $\ldots$ \\
\hline SDSS J145240.53+544345.1 & 1.520 & 18.39 & 1.35 & 1.58 & $8 \mathrm{k}(V), \mathrm{Te}(I)$ & $\cdots$ & QSO+galaxy & $\cdots$ \\
\hline SDSS J145356.57-025151.8 & 1.740 & 16.40 & 0.41 & 1.71 & $8 \mathrm{k}(V)$ & $\cdots$ & Single QSO & $\cdots$ \\
\hline SDSS J150835.59+503820.8 & 0.668 & 18.85 & 0.40 & 1.33 & $8 \mathrm{k}(V)$ & $\ldots$ & Single QSO & $\cdots$ \\
\hline SDSS J151236.95+553901.0 & 1.361 & 18.92 & 1.96 & 0.89 & $\ldots$ & MS & QSO+star & 2 \\
\hline SDSS J151304.35+021603.8 & 0.637 & 18.42 & 0.57 & 2.09 & $8 \mathrm{k}(V)$ & $\cdots$ & Single QSO & $\cdots$ \\
\hline SDSS J152445.63+440949.6 & 1.210 & 18.76 & 1.13 & 0.78 & $\mathrm{Op}(V, R, I)$ & FO & SDSS Lens & 9 \\
\hline SDSS J154120.15+542500.6 & 1.654 & 18.40 & 0.70 & 2.15 & $8 \mathrm{k}(V)$ & $\cdots$ & Single QSO & $\cdots$ \\
\hline SDSS J162935.01-010538.6 & 1.053 & 16.99 & 0.44 & 1.25 & $8 \mathrm{k}(V)$ & $\cdots$ & Single QSO & $\cdots$ \\
\hline SDSS J163348.98+313411.8 & 1.519 & 16.89 & 0.71 & 1.86 & $\ldots$ & $\ldots$ & Known lens (FBQ 1633) & 10 \\
\hline SDSS J170339.54+325957.1 & 1.375 & 17.95 & 1.41 & 2.04 & $8 \mathrm{k}(V), \mathrm{Te}(I)$ & $\cdots$ & QSO+galaxy & $\cdots$ \\
\hline SDSS J171101.70+292950.9 & 1.329 & 17.84 & 2.18 & 0.71 & $8 \mathrm{k}(I)$ & $\cdots$ & No lensing object & $\cdots$ \\
\hline SDSS J171117.66+584123.8 & 0.616 & 18.35 & 0.55 & 0.99 & $8 \mathrm{k}(V)$ & $\cdots$ & Single QSO & $\cdots$ \\
\hline SDSS J171925.07+271338.0 & 1.912 & 18.55 & 1.53 & 0.03 & $\operatorname{MM}(z)$ & $\cdots$ & No lensing object & $\cdots$ \\
\hline SDSS J172308.15+524455.5 & 1.818 & 17.14 & 1.02 & 0.88 & $\ldots$ & MS & QSO+star & 2 \\
\hline SDSS J211108.95+110642.3 & 1.003 & 19.00 & 0.62 & 0.03 & $8 \mathrm{k}(V), \mathrm{UF}(K)$ & $\cdots$ & $\theta=0 . ' 6$ & $\cdots$ \\
\hline SDSS J212243.01-002653.6 & 1.971 & 18.64 & 0.75 & 0.75 & $8 \mathrm{k}(V), \mathrm{UF}(K)$ & $\cdots$ & $\theta=0.3$ & $\cdots$ \\
\hline SDSS J213552.95-081048.1 & 0.637 & 19.10 & 0.54 & 0.35 & $8 \mathrm{k}(V)$ & $\ldots$ & Single QSO & $\cdots$ \\
\hline SDSS J213932.17-011405.7 & 1.232 & 19.03 & 0.49 & 1.05 & $\mathrm{WF}(i)$ & $\cdots$ & Single QSO & $\ldots$ \\
\hline SDSS J221110.98-000953.3 & 0.666 & 18.63 & 0.81 & 1.15 & $\mathrm{UF}(K)$ & $\cdots$ & $\theta=0.7$ & $\cdots$ \\
\hline SDSS J221729.41-081154.9 & 1.010 & 18.99 & 0.61 & 2.18 & $\mathrm{UF}(K)$ & $\cdots$ & Single QSO & $\ldots$ \\
\hline SDSS J231116.97-103849.7 & 1.540 & 18.79 & 1.81 & 1.43 & $\ldots$ & $\cdots$ & QSO+star & 2 \\
\hline SDSS J233713.66+005610.8 & 0.708 & 18.65 & 1.38 & 1.31 & $\mathrm{WF}(i)$ & WF & QSO+galaxy & $\cdots$ \\
\hline
\end{tabular}

Notes.

${ }^{a}$ Redshifts from the SDSS DR3 quasar catalog.

${ }^{\mathrm{b}} i$-band PSF magnitudes with Galactic extinction corrections from the SDSS DR3 quasar catalog.

${ }^{c}$ Image separations $\left(\theta_{\text {SDSS }}\right)$ in units of arcsec and magnitude differences $(\Delta i)$ between the expected two components, derived from fitting the SDSS $i$-band image with two PSFs using GALFIT.

d Instruments (and filters) used for the follow-up observations. 8k: UH8k at UH88, QU: QUIRC at UH88, Op: Optic at UH88, WF: WFGS2 at UH88, Te: Tek2048 at UH88, DA: DIS III at ARC 3.5 m, ES: ESI at Keck, LR: LRIS at Keck, NR: NIRC at Keck, UF: UFTI at UKIRT, FO: FOCAS at Subaru, SC: Suprime-Cam at Subaru, Ma: MagIC at WB 6.5 m, DW: Double Imaging Spectrograph at WB 6.5 m, AC: ACS at HST, NM: NICMOS at HST, RE: RETROCAM at MDM 2.4 m, MS: MMT spectrograph, AR: ARIES at MMT, EF: EFOSC2 at ESO 3.6 m, EM: EMMI at NTT, MM: MiniMo at WIYN.

References (1) Inada et al. 2005; (2) Pindor et al. 2006; (3) Oscoz et al. 1997; (4) Inada et al. 2003a; (5) Hennawi et al. 2006; (6) Inada et al. 2007b; (7) Morokuma et al. 2007; (8) Oguri et al. 2004b; (9) Oguri et al. 2008b; (10) Morgan et al. 2001.

to conclude whether they are lensed quasars or not. See Tables 2 and 3 for more details of these objects.

SDSS J0847-0013. This is a small separation $\left(\theta=1^{\prime \prime} .0\right)$ lens candidate discovered by the morphological selection criterion. Spectroscopic observations conducted with LRIS at Keck revealed that this is a quasar pair with similar redshifts of $z=0.626$ and $z=0.627$. The spectra of the two quasar components, however, have different $\mathrm{Mg}$ II emission line shapes (upper left panel of Figure 3), supporting the binary interpretation of this system. The image taken with Tek $2 \mathrm{k}$ at UH88 shows extended emission in the vicinity of the brighter component. The spectrum of this emission taken with LRIS at Keck indicates that this emission is due to the host galaxy of the quasar (Gregg et al. 2007).

SDSS J0909+5801. The spectroscopic observation at ARC $3.5 \mathrm{~m}$ revealed that both the components separated by $\theta=8^{\prime \prime} .1$ are quasars at same redshifts $(z=1.712)$. However, we see a broad absorption line feature only in the C IV emission line of the brighter component (upper right panel of Figure 3). Together with the absence of the lensing object in the deep $i$-band image, we conclude that the system is a binary quasar rather than a lens.

SDSS J0932+0722. A morphological candidate with an image separation of $\theta=1$ ". 4 found to be a pair of quasars at $z=1.994$ in Keck ESI observations. None of the deep optical and nearinfrared images shows any lensing galaxy between the two components, and the flux ratio of the two quasars in the optical is significantly different from that in the near-infrared $(0.12$ in the $I$-band and 0.02 in the $H$-band). Thus, we regard this system as a binary quasar.

SDSS J1008+0351. We detected possible extended emission between the stellar components in both deep optical and infrared images. However, spectra of this small-separation $\left(\theta=1^{\prime \prime}\right.$. 1$)$ candidate taken with FOCAS at Subaru suggest that this is a binary quasar, because of the slightly different redshifts $(z=1.745$ and 1.740) and the different overall shapes of the spectra, as shown in the lower left panel of Figure 3. One interpretation is that the extended emission is due to the host galaxy of one (or both) of the quasars.

SDSS J2111+1050. This is a large separation $(\theta=9$ '.7) lens candidate from the color-selected sample. From ARC $3.5 \mathrm{~m}$ spectroscopy, the redshifts of the two stellar components are quite similar with $z=1.897$. However, the SEDs (in particular, the shapes of the $C$ IV emission lines; see the lower right panel of Figure 3) are different, and a deep I-band image does not show a lensing galaxy between the two components. We conclude that this is a binary quasar rather than a lens. 
Table 3

Color Candidates

\begin{tabular}{|c|c|c|c|c|c|c|c|}
\hline Object & $z^{\mathrm{a}}$ & $i_{\text {cor }}^{\mathrm{b}}$ & $\theta_{\text {SDSS }^{c}}$ & Image $^{\mathrm{d}}$ & $\operatorname{Spec}^{\mathrm{d}}$ & Comment & Ref. \\
\hline SDSS J004757.25+144741.9 & 1.612 & 18.67 & & & & & \\
\hline SDSS J004757.87+144744.7 & $(2.790)$ & 18.72 & 9.42 & $\cdots$ & DA & QSO pair & $\cdots$ \\
\hline SDSS J021649.25-003723.5 & 1.542 & 18.55 & & & & & \\
\hline SDSS J021649.15-003711.5 & $\cdots$ & 19.57 & 12.15 & $\cdots$ & DA & QSO+star & $\cdots$ \\
\hline SDSS J023205.09+010640.2 & 1.259 & 17.28 & & & & & \\
\hline SDSS J023205.18+010634.2 & $\ldots$ & 17.90 & 6.20 & $\cdots$ & DA & QSO+star & $\cdots$ \\
\hline SDSS J025804.27-001059.9 & 1.551 & 18.93 & & & & & \\
\hline SDSS J025803.81-001118.1 & $\ldots$ & 20.05 & 19.43 & $\cdots$ & DA & Different SED & $\cdots$ \\
\hline SDSS J073118.72+371100.6 & 1.468 & 18.96 & & & & & \\
\hline SDSS J073119.02+371115.4 & $\cdots$ & 18.75 & 15.21 & $\cdots$ & DA & QSO+star & $\cdots$ \\
\hline SDSS J074013.44+292648.4 & 0.980 & 18.42 & & & & & \\
\hline SDSS J074013.42+292645.8 & $(0.978)$ & 19.68 & 2.64 & $\cdots$ & $\ldots$ & QSO pair & 1 \\
\hline SDSS J074357.07+300742.6 & 2.177 & 18.82 & & & & & \\
\hline SDSS J074355.82+300731.1 & $\ldots$ & 18.59 & 19.88 & $\cdots$ & DA & QSO+star & $\cdots$ \\
\hline SDSS J075011.23+430419.0 & 1.265 & 18.87 & & & & & \\
\hline SDSS J075011.66+430413.3 & $\ldots$ & 18.87 & 7.37 & $\cdots$ & DA & QSO+star & $\cdots$ \\
\hline SDSS J075310.75+292110.4 & 1.937 & 18.84 & & & & & \\
\hline SDSS J075311.03+292055.0 & $\cdots$ & 19.60 & 15.77 & $\cdots$ & DA & QSO+star & $\cdots$ \\
\hline SDSS J080435.00+314311.4 & 2.141 & 19.05 & & & & & \\
\hline SDSS J080435.23+314328.7 & & 19.74 & 17.53 & $\cdots$ & DA & Different SED & $\ldots$ \\
\hline SDSS J080658.22+273448.0 & 1.243 & 18.63 & & & & & \\
\hline SDSS J080658.17+273450.1 & $\ldots$ & 19.91 & 2.26 & $\mathrm{WF}(i)$ & $\cdots$ & No lensing object & $\cdots$ \\
\hline SDSS J081502.51+262804.2 & 1.286 & 18.56 & & & & & \\
\hline SDSS J081502.28+262804.7 & $\cdots$ & 19.53 & 3.11 & $\cdots$ & DA & Different SED & $\cdots$ \\
\hline SDSS J081617.73+293639.6 & 0.768 & 18.24 & & & & & \\
\hline SDSS J081618.06+293643.7 & $\ldots$ & 19.51 & 5.92 & $\mathrm{UF}(K)$ & $\ldots$ & No lensing object & $\cdots$ \\
\hline SDSS J081624.72+324928.9 & 1.259 & 18.59 & & & & & \\
\hline SDSS J081624.08+324931.0 & $\ldots$ & 18.39 & 8.44 & $\cdots$ & DA & QSO+star & $\cdots$ \\
\hline SDSS J081812.94+511923.4 & 2.098 & 18.98 & & & & & \\
\hline SDSS J081813.02+511917.5 & $\ldots$ & 19.80 & 6.05 & $\cdots$ & DA & Different SED & $\cdots$ \\
\hline SDSS J081904.56+035455.6 & 0.886 & 18.44 & & & & & \\
\hline SDSS J081904.40+035455.8 & $\cdots$ & 19.66 & 2.41 & $\mathrm{WF}(i)$ & $\ldots$ & No lensing object & $\cdots$ \\
\hline SDSS J082046.24+035742.1 & 1.574 & 18.57 & & & & & \\
\hline SDSS J082046.67+035740.9 & $(1.870)$ & 19.13 & 6.44 & $\cdots$ & DA & QSO pair & $\cdots$ \\
\hline SDSS J083216.99+040405.2 & 1.115 & 18.89 & & & & & \\
\hline SDSS J083217.11+040403.8 & (1.115) & 19.97 & 2.23 & $\mathrm{Te}(V, I), \mathrm{UF}(K)$ & $\mathrm{EF}$ & SDSS lens & 2 \\
\hline SDSS J083349.46+440952.7 & 2.011 & 19.03 & & & & & \\
\hline SDSS J083349.19+440954.0 & $\cdots$ & 19.89 & 3.17 & $\mathrm{UF}(K)$ & $\cdots$ & No lensing object & $\cdots$ \\
\hline SDSS J083557.50+341455.4 & 1.328 & 18.91 & & & & & \\
\hline SDSS J083556.32+341504.2 & $(1.505)$ & 19.44 & 17.08 & $\mathrm{UF}(K)$ & DA & QSO pair & $\cdots$ \\
\hline SDSS J083649.55+484154.0 & 1.710 & 18.04 & & & & & \\
\hline SDSS J083649.45+484150.0 & $(0.657)$ & 19.09 & 4.10 & $\cdots$ & $\cdots$ & QSO pair & 1 \\
\hline SDSS J084137.51+032830.0 & 1.585 & 19.00 & & & & & \\
\hline SDSS J084137.75+032837.1 & $\ldots$ & 19.88 & 7.93 & $\ldots$ & DA & QSO+star & $\cdots$ \\
\hline SDSS J090809.13+444138.9 & 1.722 & 17.78 & & & & & \\
\hline SDSS J090809.45+444158.6 & $\cdots$ & 18.70 & 20.03 & $\cdots$ & DA & QSO+star & $\cdots$ \\
\hline SDSS J090955.54+580143.2 & 1.712 & 18.97 & & & & & \\
\hline SDSS J090956.50+580140.5 & $(1.712)$ & 20.18 & 8.07 & $\mathrm{WF}(i)$ & DA & QSO pair, no lensing object & $\cdots$ \\
\hline SDSS J092024.21+030636.0 & 1.363 & 18.86 & & & & & \\
\hline SDSS J092024.01+030650.9 & $(1.450)$ & 19.56 & 15.20 & $\ldots$ & DA & QSO pair & $\cdots$ \\
\hline SDSS J092151.55+524559.7 & 1.991 & 18.98 & & & & & \\
\hline SDSS J092153.00+524551.4 & $\ldots$ & 20.17 & 15.59 & $\cdots$ & DA & Different SED & $\cdots$ \\
\hline SDSS J092248.35+515611.8 & 1.825 & 18.49 & & & & & \\
\hline SDSS J092246.22+515614.5 & $\cdots$ & 18.23 & 19.82 & $\cdots$ & DA & QSO+star & $\cdots$ \\
\hline SDSS J093336.57+620521.8 & 2.145 & 19.10 & & & & & \\
\hline SDSS J093336.59+620509.3 & $\cdots$ & 19.52 & 12.55 & $\cdots$ & DA & QSO+star & $\cdots$ \\
\hline SDSS J094309.66+103400.6 & 1.239 & 18.62 & & & & & \\
\hline SDSS J094309.36+103401.3 & $(1.430)$ & 19.60 & 4.57 & $\cdots$ & $\mathrm{EF}$ & QSO pair & $\cdots$ \\
\hline SDSS J094510.75+472448.8 & 1.952 & 18.90 & & & & & \\
\hline SDSS J094510.88+472435.9 & $(0.560)$ & 20.02 & 12.92 & $\cdots$ & DA & QSO pair & $\cdots$ \\
\hline SDSS J095224.84+064732.0 & 2.186 & 18.08 & & & & & \\
\hline SDSS J095225.25+064742.8 & $\cdots$ & 18.50 & 12.41 & $\cdots$ & DA & QSO+star & $\cdots$ \\
\hline SDSS J095711.08+640548.6 & 1.338 & 18.62 & & & & & \\
\hline
\end{tabular}


Table 3

(Continued)

\begin{tabular}{|c|c|c|c|c|c|c|c|}
\hline Object & $z^{\mathrm{a}}$ & $i_{\text {cor }}{ }^{b}$ & $\theta_{\text {SDSS }^{c}}$ & Image $^{\mathrm{d}}$ & Spec $^{\mathrm{d}}$ & Comment & Ref. \\
\hline SDSS J095711.22+640602.7 & $(1.288)$ & 19.86 & 14.11 & $\cdots$ & DA & QSO pair & $\ldots$ \\
\hline SDSS J095841.15+385629.9 & 1.639 & 18.94 & & & & & \\
\hline SDSS J095839.67+385621.9 & $\ldots$ & 18.99 & 18.98 & $\ldots$ & DA & Different SED & $\cdots$ \\
\hline SDSS J100034.17+540628.6 & 1.212 & 18.64 & & & & & \\
\hline SDSS J100034.86+540641.4 & $(1.220)$ & 19.14 & 14.19 & $\mathrm{Te}(I)$ & DA & QSO pair, no lensing object & .. \\
\hline SDSS J100120.84+555349.5 & 1.413 & 16.67 & & & & & \\
\hline SDSS J100120.69+555355.6 & $(1.405)$ & 16.81 & 6.16 & $\ldots$ & $\cdots$ & Known lens (Q0957) & 4 \\
\hline SDSS J100128.61+502756.8 & 1.839 & 17.31 & & & & & \\
\hline SDSS J100128.35+502758.4 & $(1.838)$ & 17.68 & 2.93 & $8 \mathrm{k}(V, R, I), \operatorname{SP}(z)$ & DA & SDSS Lens & 3 \\
\hline SDSS J100304.55+444335.9 & 1.630 & 18.80 & & & & & \\
\hline SDSS J100304.61+444338.8 & $\ldots$ & 19.93 & 2.95 & $8 \mathrm{k}(V, I)$ & $\cdots$ & No lensing object & $\cdots$ \\
\hline SDSS J100434.91+411242.8 & 1.740 & 18.84 & & & & & \\
\hline SDSS J100434.80+411239.2 & (1.734) & 18.41 & 3.76 & $\mathrm{SC}(g, r, i, z), \operatorname{SP}(r)$ & DA, LR & SDSS Lens (component A) & 5,6 \\
\hline SDSS J100433.82+411234.8 & (1.734) & 19.34 & 14.71 & $\mathrm{SC}(g, r, i, z), \operatorname{SP}(r)$ & DA, LR & SDSS Lens (component C) & 5,6 \\
\hline SDSS J101930.41+522411.7 & 1.961 & 18.24 & & & & & \\
\hline SDSS J101930.17+522413.2 & $\ldots$ & 19.47 & 2.70 & $8 \mathrm{k}(V), \mathrm{Op}(I)$ & $\cdots$ & No lensing object & $\cdots$ \\
\hline SDSS J103423.11+623340.3 & 1.406 & 18.76 & & & & & \\
\hline SDSS J103423.16+623343.3 & $\ldots$ & 19.86 & 3.03 & $8 \mathrm{k}(V), \mathrm{Op}(I)$ & $\cdots$ & No lensing object & $\cdots$ \\
\hline SDSS J103519.36+075258.0 & 1.215 & 19.03 & & & & & \\
\hline SDSS J103519.22+075256.3 & $(1.218)$ & 20.11 & 2.66 & $8 \mathrm{k}(I), \mathrm{QU}(H)$ & $\cdots$ & QSO pair, no lensing object & 1 \\
\hline SDSS J103716.94+014126.3 & 1.576 & 19.05 & & & & & \\
\hline SDSS J103717.79+014121.0 & $\ldots$ & 18.68 & 13.95 & $\cdots$ & DA & QSO+star & $\ldots$ \\
\hline SDSS J103724.73+580513.0 & 1.517 & 17.39 & & & & & \\
\hline SDSS J103724.20+580514.1 & $\ldots$ & 17.99 & 4.36 & $\cdots$ & DA & QSO+star & $\cdots$ \\
\hline SDSS J103950.00+593511.6 & 1.560 & 18.92 & & & & & \\
\hline SDSS J103949.51+593505.9 & $\ldots$ & 19.72 & 6.81 & $\cdots$ & DA & Different SED & $\cdots$ \\
\hline SDSS J104213.61+061942.0 & 1.559 & 18.67 & & & & & \\
\hline SDSS J104214.22+061959.1 & $(1.620)$ & 19.60 & 19.41 & $\ldots$ & DA & QSO pair & $\ldots$ \\
\hline SDSS J104658.02+471726.9 & 1.532 & 18.58 & & & & & \\
\hline SDSS J104657.05+471737.4 & $(2.060)$ & 19.24 & 14.43 & $\cdots$ & DA & QSO pair & $\cdots$ \\
\hline SDSS J110536.22+031952.4 & 0.922 & 18.15 & & & & & \\
\hline SDSS J110536.29+031955.3 & $\ldots$ & 18.92 & 3.06 & $\cdots$ & DA & Different SED & $\cdots$ \\
\hline SDSS J110932.13+531635.7 & 0.982 & 18.72 & & & & & \\
\hline SDSS J110932.49+531635.5 & $(1.350)$ & 19.00 & 3.25 & $\cdots$ & DA & QSO pair & $\cdots$ \\
\hline SDSS J112456.26+090848.8 & 1.649 & 19.03 & & & & & \\
\hline SDSS J112455.65+090849.7 & $\ldots$ & 19.43 & 9.16 & $\mathrm{Op}(I)$ & $\cdots$ & No lensing object & $\cdots$ \\
\hline SDSS J113813.76+024548.4 & 1.639 & 18.85 & & & & & \\
\hline SDSS J113814.17+024556.3 & $\ldots$ & 17.64 & 10.10 & $\cdots$ & DA & QSO+star & $\cdots$ \\
\hline SDSS J114306.19+025402.1 & 1.605 & 19.02 & & & & & \\
\hline SDSS J114305.85+025413.1 & $\ldots$ & 19.89 & 12.14 & $\mathrm{Op}(I)$ & $\cdots$ & No lensing object & $\cdots$ \\
\hline SDSS J114546.22+032251.9 & 2.008 & 19.01 & & & & & \\
\hline SDSS J114546.54+032236.7 & (1.773) & 19.94 & 15.93 & $\cdots$ & $\cdots$ & QSO pair & 1 \\
\hline SDSS J115940.79-003203.5 & 2.033 & 17.62 & & & & & \\
\hline SDSS J115940.40-003213.9 & $\cdots$ & 18.68 & 11.83 & $\cdots$ & DA & Different SED & $\cdots$ \\
\hline SDSS J120457.12+043241.0 & 1.187 & 18.28 & & & & & \\
\hline SDSS J120456.64+043245.7 & $\ldots$ & 19.50 & 8.55 & $\mathrm{Op}(I)$ & $\cdots$ & No lensing object & $\cdots$ \\
\hline SDSS J120523.68-033618.2 & 1.495 & 18.80 & & & & & \\
\hline SDSS J120522.75-033614.6 & $\ldots$ & 17.54 & 14.32 & $\cdots$ & DA & QSO+star & $\cdots$ \\
\hline SDSS J121002.47+495312.7 & 1.618 & 18.96 & & & & & \\
\hline SDSS J121002.28+495253.1 & $(1.554)$ & 20.06 & 19.74 & $\mathrm{Op}(I)$ & DA & QSO pair, no lensing object & $\ldots$ \\
\hline SDSS J121636.02+543159.2 & 1.894 & 19.01 & & & & & \\
\hline SDSS J121637.35+543158.2 & (1.780) & 20.03 & 11.57 & $\cdots$ & DA & QSO pair & $\cdots$ \\
\hline SDSS J121647.22+495720.4 & 1.200 & 18.34 & & & & & \\
\hline SDSS J121647.62+495710.6 & $(1.195)$ & 19.55 & 10.53 & $\mathrm{Op}(I)$ & DA & QSO pair, no lensing object & $\cdots$ \\
\hline SDSS J122126.47+572414.0 & 1.959 & 18.99 & & & & & \\
\hline SDSS J122126.69+572416.6 & $\ldots$ & 20.27 & 3.12 & $8 \mathrm{k}(V), \mathrm{Op}(I)$ & $\cdots$ & No lensing object & $\cdots$ \\
\hline SDSS J123504.17+020743.7 & 2.169 & 18.77 & & & & & \\
\hline SDSS J123505.16+020734.8 & $\ldots$ & 19.51 & 17.31 & $\ldots$ & DA & QSO+star & $\cdots$ \\
\hline SDSS J123558.55-023503.3 & 2.064 & 18.36 & & & & & \\
\hline SDSS J123558.36-023503.6 & $\ldots$ & 17.69 & 2.80 & $\cdots$ & DA & QSO+star & $\cdots$ \\
\hline SDSS J124315.47-012120.8 & 1.864 & 18.70 & & & & & \\
\hline SDSS J124314.68-012135.5 & $\ldots$ & 18.83 & 18.88 & $\mathrm{Op}(I)$ & DA & QSO+star & $\cdots$ \\
\hline SDSS J125029.25+025747.9 & 0.610 & 18.25 & & & & & \\
\hline SDSS J125028.62+025745.1 & $\ldots$ & 17.11 & 9.86 & $\cdots$ & DA & QSO+star & $\cdots$ \\
\hline
\end{tabular}


Table 3

(Continued)

\begin{tabular}{|c|c|c|c|c|c|c|c|}
\hline Object & $z^{\mathrm{a}}$ & $i_{\text {cor }}{ }^{b}$ & $\theta_{\text {SDSS }^{c}}$ & Image $^{\mathrm{d}}$ & Spec $^{\mathrm{d}}$ & Comment & Ref. \\
\hline SDSS J125422.00+610421.6 & 2.055 & 18.91 & & & & & \\
\hline SDSS J125420.54+610435.6 & $(2.041)$ & 19.27 & 17.61 & $\cdots$ & $\cdots$ & QSO pair & 1 \\
\hline SDSS J131008.97+660350.3 & 1.747 & 18.90 & & & & & \\
\hline SDSS J131008.54+660349.6 & $\cdots$ & 20.04 & 2.74 & $8 \mathrm{k}(I)$ & $\cdots$ & No lensing object & $\cdots$ \\
\hline SDSS J131505.88+590157.5 & 1.933 & 17.50 & & & & & \\
\hline SDSS J131507.15+590150.7 & $\ldots$ & 17.59 & 11.89 & $\ldots$ & DA & QSO+star & $\cdots$ \\
\hline SDSS J132732.66-031645.7 & 1.274 & 19.06 & & & & & \\
\hline SDSS J132733.00-031644.3 & $\ldots$ & 19.85 & 5.31 & $\ldots$ & DA & Different SED & $\cdots$ \\
\hline SDSS J133303.35+000451.3 & 1.511 & 19.05 & & & & & \\
\hline SDSS J133302.65+000437.8 & $\ldots$ & 18.07 & 17.14 & $\cdots$ & DA & QSO+star & $\cdots$ \\
\hline SDSS J133945.37+000946.1 & 1.872 & 18.85 & & & & & \\
\hline SDSS J133945.06+001004.4 & $(0.978)$ & 19.85 & 18.85 & $\cdots$ & $\cdots$ & QSO pair, from $2 \mathrm{dF}$ data & 7 \\
\hline SDSS J134702.84+032233.4 & 1.710 & 18.96 & & & & & \\
\hline SDSS J134703.70+032238.4 & $\cdots$ & 18.87 & 13.80 & $\mathrm{WF}(i)$ & $\cdots$ & No lensing object & $\cdots$ \\
\hline SDSS J134918.53-015734.8 & 1.586 & 19.09 & & & & & \\
\hline SDSS J134919.32-015736.9 & $\ldots$ & 19.31 & 12.01 & $\mathrm{Op}(I)$ & $\ldots$ & No lensing object & $\cdots$ \\
\hline SDSS J135418.26+585935.9 & 0.791 & 19.00 & & & & & \\
\hline SDSS J135418.10+585951.7 & $\cdots$ & 19.14 & 15.82 & $\cdots$ & DA & Different SED & $\cdots$ \\
\hline SDSS J135752.15+051544.7 & 1.595 & 18.92 & & & & & \\
\hline SDSS J135751.03+051545.5 & $\cdots$ & 20.13 & 16.78 & $\cdots$ & DA & Different SED & $\cdots$ \\
\hline SDSS J140016.87+542131.7 & 1.479 & 18.99 & & & & & \\
\hline SDSS J140016.19+542136.6 & $(1.810)$ & 19.84 & 7.72 & $\mathrm{Op}(I)$ & DA & QSO pair & $\cdots$ \\
\hline SDSS J140326.90+561307.3 & 1.075 & 17.09 & & & & & \\
\hline SDSS J140326.97+561305.5 & $\ldots$ & 18.26 & 1.95 & $8 \mathrm{k}(I)$ & $\cdots$ & QSO+galaxy+galaxy & $\cdots$ \\
\hline SDSS J142327.05+591819.8 & 1.672 & 19.06 & & & & & \\
\hline SDSS J142326.24+591833.5 & $\cdots$ & 18.25 & 15.08 & $\cdots$ & DA & QSO+star & $\cdots$ \\
\hline SDSS J142359.48+545250.8 & 1.409 & 18.31 & & & & & \\
\hline SDSS J142400.00+545248.7 & $(0.610)$ & 19.56 & 4.94 & $\cdots$ & DA & QSO pair & $\cdots$ \\
\hline SDSS J142541.12+021018.7 & 1.665 & 19.04 & & & & & \\
\hline SDSS J142542.27+021010.1 & $\cdots$ & 19.77 & 19.27 & $\mathrm{Op}(I)$ & $\cdots$ & No lensing object & $\cdots$ \\
\hline SDSS J142944.25+042202.6 & 1.148 & 18.86 & & & & & \\
\hline SDSS J142944.18+042205.6 & $\cdots$ & 19.69 & 3.20 & $\mathrm{Op}(I), 8 \mathrm{k}(I)$ & $\cdots$ & No lensing object & $\cdots$ \\
\hline SDSS J143433.45+613752.7 & 1.818 & 19.04 & & & & & \\
\hline SDSS J143432.68+613737.0 & $(0.427)$ & 19.21 & 16.59 & $\mathrm{WF}(i)$ & DA & QSO pair & $\cdots$ \\
\hline SDSS J143735.94+033334.3 & 2.122 & 18.63 & & & & & \\
\hline SDSS J143736.44+033326.9 & $\cdots$ & 19.13 & 10.55 & $\cdots$ & DA & Different SED & $\cdots$ \\
\hline SDSS J143817.62+031908.6 & 1.483 & 19.01 & & & & & \\
\hline SDSS J143817.02+031901.9 & $\cdots$ & 20.27 & 11.24 & $\cdots$ & DA & QSO+star & $\cdots$ \\
\hline SDSS J144104.91+044348.4 & 1.112 & 18.42 & & & & & \\
\hline SDSS J144104.58+044350.8 & $\cdots$ & 18.69 & 5.48 & $\mathrm{WF}(i)$ & $\cdots$ & No lensing object & $\cdots$ \\
\hline SDSS J144145.09+023743.0 & 1.160 & 19.07 & & & & & \\
\hline SDSS J144145.09+023744.8 & $\cdots$ & 19.46 & 1.75 & $\cdots$ & MS & QSO+star & $\cdots$ \\
\hline SDSS J145455.89+420306.0 & 1.598 & 19.01 & & & & & \\
\hline SDSS J145455.73+420256.1 & $\cdots$ & 19.60 & 10.04 & $\mathrm{WF}(i)$ & DA & QSO+star & $\cdots$ \\
\hline SDSS J150319.42+475206.8 & 0.757 & 17.92 & & & & & \\
\hline SDSS J150319.46+475209.7 & $\cdots$ & 18.74 & 2.84 & $8 \mathrm{k}(I)$ & $\cdots$ & No lensing object & $\cdots$ \\
\hline SDSS J150503.46-022324.4 & 2.097 & 18.61 & & & & & \\
\hline SDSS J150503.70-022308.6 & $\cdots$ & 19.21 & 16.23 & $\cdots$ & DA & QSO+star & $\cdots$ \\
\hline SDSS J150533.86+013201.8 & 1.512 & 18.67 & & & & & \\
\hline SDSS J150534.19+013145.7 & $\cdots$ & 18.07 & 16.84 & $\cdots$ & DA & QSO+star & $\cdots$ \\
\hline SDSS J151519.40-001103.4 & 1.564 & 19.01 & & & & & \\
\hline SDSS J151519.94-001049.3 & $\cdots$ & 19.45 & 16.36 & $\mathrm{SC}(I)$ & $\cdots$ & No lensing object & $\cdots$ \\
\hline SDSS J152130.70+023915.1 & 2.127 & 18.71 & & & & & \\
\hline SDSS J152129.83+023917.9 & $\ldots$ & 19.08 & 13.33 & $\cdots$ & DA & QSO+star & $\cdots$ \\
\hline SDSS J152510.00+602828.1 & 1.337 & 19.03 & & & & & \\
\hline SDSS J152510.45+602833.3 & $\cdots$ & 19.25 & 6.19 & $\mathrm{Op}(I)$ & $\cdots$ & No lensing object & $\cdots$ \\
\hline SDSS J153518.78+582912.2 & 1.527 & 19.06 & & & & & \\
\hline SDSS J153518.86+582925.9 & $\cdots$ & 19.62 & 13.70 & $\mathrm{Op}(I)$ & DA & QSO+star & $\cdots$ \\
\hline SDSS J153559.97+430819.0 & 1.620 & 18.67 & & & & & \\
\hline SDSS J153559.26+430833.1 & $\cdots$ & 18.57 & 16.01 & $\cdots$ & DA & QSO+star & $\cdots$ \\
\hline SDSS J154107.47-003716.0 & 0.755 & 17.57 & & & & & \\
\hline SDSS J154107.21-003711.8 & $\cdots$ & 18.07 & 5.65 & $\cdots$ & MS & QSO+star & 8 \\
\hline SDSS J154148.81+523051.9 & 2.054 & 19.01 & & & & & \\
\hline SDSS J154148.93+523053.6 & $\cdots$ & 19.59 & 1.94 & $8 \mathrm{k}(I)$ & $\cdots$ & No lensing object & $\cdots$ \\
\hline SDSS J155723.93+492607.5 & 2.195 & 18.42 & & & & & \\
\hline
\end{tabular}


Table 3

(Continued)

\begin{tabular}{|c|c|c|c|c|c|c|c|}
\hline Object & $z^{\mathrm{a}}$ & $i_{\text {cor }}^{b}$ & $\theta_{\text {SDSS }^{c}}$ & Image $^{\mathrm{d}}$ & $\mathrm{Spec}^{\mathrm{d}}$ & Comment & Ref. \\
\hline SDSS J155723.79+492551.1 & $\ldots$ & 19.69 & 16.46 & $\ldots$ & DA & Different SED & $\ldots$ \\
\hline SDSS J160305.94+272100.9 & 1.620 & 18.14 & & & & & \\
\hline SDSS J160307.00+272059.3 & $\ldots$ & 18.43 & 14.21 & $\cdots$ & DA & QSO+star & $\cdots$ \\
\hline SDSS J160547.59+511330.2 & 1.785 & 19.09 & & & & & \\
\hline SDSS J160546.66+511322.6 & $(1.844)$ & 18.46 & 11.63 & $\cdots$ & $\cdots$ & QSO pair & 1 \\
\hline SDSS J160914.86+380728.1 & 1.126 & 18.64 & & & & & \\
\hline SDSS J160914.85+380730.0 & $\ldots$ & 18.68 & 1.93 & $8 \mathrm{k}(I)$ & $\ldots$ & No lensing object & $\cdots$ \\
\hline SDSS J161953.24+351321.8 & 1.901 & 18.64 & & & & & \\
\hline SDSS J161953.45+351323.5 & $\cdots$ & 19.50 & 3.13 & $8 \mathrm{k}(I)$ & $\cdots$ & No lensing object & $\cdots$ \\
\hline SDSS J161952.82+351315.4 & $\ldots$ & 19.89 & 8.20 & $8 \mathrm{k}(I)$ & $\cdots$ & No lensing object & $\cdots$ \\
\hline SDSS J162602.40+334030.0 & 1.541 & 18.77 & & & & & \\
\hline SDSS J162603.61+334025.9 & $\cdots$ & 19.97 & 15.65 & $\mathrm{WF}(i)$ & $\cdots$ & No lensing object & $\cdots$ \\
\hline SDSS J162902.59+372430.8 & 0.926 & 19.03 & & & & & \\
\hline SDSS J162902.63+372435.1 & $(0.906)$ & 19.37 & 4.35 & $\mathrm{NR}\left(K^{\prime}\right)$ & $\cdots$ & QSO pair, no lensing object & 1 \\
\hline SDSS J165248.93+352134.1 & 1.498 & 18.33 & & & & & \\
\hline SDSS J165250.29+352141.6 & $\cdots$ & 19.20 & 18.26 & $\mathrm{WF}(i)$ & $\cdots$ & No lensing object & $\cdots$ \\
\hline SDSS J165459.71+305208.3 & 1.971 & 19.08 & & & & & \\
\hline SDSS J165459.12+305158.5 & $\cdots$ & 19.41 & 12.36 & $\ldots$ & DA & QSO+star & $\cdots$ \\
\hline SDSS J171334.41+553050.3 & 1.276 & 18.54 & & & & & \\
\hline SDSS J171335.03+553047.9 & $\cdots$ & 18.59 & 5.84 & $\cdots$ & DA & QSO+star & $\cdots$ \\
\hline SDSS J172633.52+530300.4 & 0.654 & 19.00 & & & & & \\
\hline SDSS J172633.44+530302.0 & $\cdots$ & 19.07 & 1.70 & $8 \mathrm{k}(I)$ & $\cdots$ & No lensing object & $\cdots$ \\
\hline SDSS J172806.78+582039.2 & 2.011 & 19.06 & & & & & \\
\hline SDSS J172808.74+582040.0 & $\ldots$ & 20.17 & 15.46 & $\mathrm{WF}(i)$ & DA & QSO+star & $\cdots$ \\
\hline SDSS J203845.35+005532.1 & 1.903 & 18.53 & & & & & \\
\hline SDSS J203846.08+005541.4 & $\cdots$ & 19.47 & 14.32 & $\mathrm{WF}(i)$ & DA & No lensing object, different SED & $\cdots$ \\
\hline SDSS J203955.67-054102.8 & 1.460 & 18.90 & & & & & \\
\hline SDSS J203955.63-054044.7 & $\cdots$ & 19.80 & 18.08 & $\mathrm{WF}(i)$ & $\cdots$ & No lensing object & $\cdots$ \\
\hline SDSS J204030.52-003015.9 & 1.549 & 18.84 & & & & & \\
\hline SDSS J204030.71-003010.5 & $\cdots$ & 18.33 & 6.04 & $\mathrm{WF}(i)$ & $\cdots$ & No lensing object & $\cdots$ \\
\hline SDSS J204113.41-060158.5 & 1.432 & 18.63 & & & & & \\
\hline SDSS J204114.12-060149.0 & 0.830 & 19.56 & 14.19 & $\mathrm{Op}(I)$ & DA & QSO pair & $\cdots$ \\
\hline SDSS J210706.22-062506.7 & 1.577 & 18.91 & & & & & \\
\hline SDSS J210706.31-062503.2 & $\cdots$ & 19.56 & 3.71 & $\mathrm{WF}(i)$ & $\cdots$ & No lensing object & $\cdots$ \\
\hline SDSS J210946.73+105601.0 & 1.628 & 18.57 & & & & & \\
\hline SDSS J210946.23+105559.5 & $\cdots$ & 18.39 & 7.53 & $\cdots$ & DA & QSO+star & $\cdots$ \\
\hline SDSS J211102.60+105038.3 & 1.897 & 18.87 & & & & & \\
\hline SDSS J211102.41+105047.5 & $(1.897)$ & 19.02 & 9.67 & $\mathrm{Te}(I)$ & DA & QSO pair (different SED) & \\
\hline SDSS J211230.33-063332.1 & 1.554 & 18.94 & & & & & \\
\hline SDSS J211229.31-063331.4 & $(0.551)$ & 19.71 & 15.21 & $\cdots$ & $\cdots$ & QSO pair & 1 \\
\hline SDSS J211610.83+111429.0 & 1.076 & 18.46 & & & & & \\
\hline SDSS J211610.58+111423.2 & $\cdots$ & 19.23 & 6.83 & $\cdots$ & DA & Different SED & $\cdots$ \\
\hline SDSS J212429.83-004727.0 & 1.614 & 18.96 & & & & & \\
\hline SDSS J212430.90-004725.2 & $\cdots$ & 19.11 & 16.26 & $\cdots$ & DA & Different SED & $\cdots$ \\
\hline SDSS J212906.23-071613.3 & 1.213 & 18.57 & & & & & \\
\hline SDSS J212906.32-071612.1 & $\cdots$ & 17.81 & 1.79 & $\cdots$ & $\cdots$ & QSO+star & 8 \\
\hline SDSS J212956.44-005150.4 & 1.112 & 18.98 & & & & & \\
\hline SDSS J212956.56-005152.4 & $\cdots$ & 20.04 & 2.68 & $\mathrm{WF}(i)$ & $\cdots$ & No lensing object & $\cdots$ \\
\hline SDSS J213414.01-004533.1 & 1.535 & 18.77 & & & & & \\
\hline SDSS J213414.17-004514.6 & $\cdots$ & 18.44 & 18.59 & $\cdots$ & DA & Different SED & $\cdots$ \\
\hline SDSS J213639.11+114731.8 & 1.518 & 19.04 & & & & & \\
\hline SDSS J213640.40+114736.9 & $\cdots$ & 18.13 & 19.47 & $\cdots$ & DA & Different SED & $\cdots$ \\
\hline SDSS J220256.32-092556.7 & 0.723 & 18.31 & & & & & \\
\hline SDSS J220257.01-092602.3 & $\cdots$ & 18.80 & 11.71 & $\cdots$ & DA & Different SED & $\cdots$ \\
\hline SDSS J222504.14+123814.5 & 1.549 & 19.04 & & & & & \\
\hline SDSS J222503.32+123815.1 & $\cdots$ & 18.15 & 12.08 & $\cdots$ & DA & QSO+star & $\cdots$ \\
\hline SDSS J222822.17-005943.5 & 2.172 & 18.62 & & & & & \\
\hline SDSS J222822.18-005949.3 & $\cdots$ & 19.14 & 5.82 & $\cdots$ & DA & QSO+star & $\cdots$ \\
\hline SDSS J223219.23+140017.0 & 0.725 & 18.87 & & & & & \\
\hline SDSS J223219.42+140012.6 & $\cdots$ & 19.06 & 5.16 & $\cdots$ & DA & QSO+star & $\cdots$ \\
\hline SDSS J230250.85+143203.2 & 0.654 & 18.99 & & & & & \\
\hline SDSS J230250.90+143158.5 & $\cdots$ & 20.17 & 4.71 & $\cdots$ & DA & Different SED & $\cdots$ \\
\hline SDSS J230951.18-094016.3 & 1.572 & 19.07 & & & & & \\
\hline SDSS J230950.59-093958.3 & $\cdots$ & 20.32 & 20.04 & $\cdots$ & DA & Different SED & $\cdots$ \\
\hline SDSS J231152.83+145455.0 & 1.261 & 18.98 & & & & & \\
\hline
\end{tabular}


Table 3

(Continued)

\begin{tabular}{|c|c|c|c|c|c|c|c|}
\hline Object & $z^{\mathrm{a}}$ & $i_{\text {cor }}{ }^{b}$ & $\theta_{\text {SDSS }}{ }^{\mathrm{c}}$ & Image $^{\mathrm{d}}$ & Spec $^{d}$ & Comment & Ref. \\
\hline SDSS J231152.38+145507.5 & .. & 19.88 & 14.17 & $\ldots$ & $\ldots$ & QSO+star & 1 \\
\hline SDSS J235108.66+134322.6 & 1.218 & 18.81 & & & & & \\
\hline SDSS J235108.70+134320.8 & $\ldots$ & 19.92 & 1.86 & $\mathrm{WF}(i)$ & .. & No lensing object & $\ldots$ \\
\hline SDSS J235924.73+152541.6 & 1.574 & 19.01 & & & & & \\
\hline SDSS J235924.84+152544.3 & $\ldots$ & 19.98 & 3.09 & $\mathrm{WF}(i)$ & $\ldots$ & No lensing object & $\ldots$ \\
\hline
\end{tabular}

Notes. Two candidates that are identified by the morphological selection algorithm as well, SDSS J073406.75+273355.6 and SDSS $\mathrm{J} 133534.79+011805.5$, are listed in Table 2.

${ }^{a}$ Redshifts from the SDSS DR3 quasar catalog and the follow-up observations (in parentheses).

${ }^{\mathrm{b}} i$-band PSF magnitudes with the Galactic extinction correction from the SDSS DR3 quasar catalog.

${ }^{\mathrm{c}}$ Image separations in units of arcsec between two components from the SDSS imaging data.

${ }^{\mathrm{d}}$ Instruments (and filters) used for the follow-up observations. 8k: UH8k at UH88, QU: QUIRC at UH88, Op: Optic at UH88, WF: WFGS2 at UH88, Te: Tek2048 at UH88, DA: DIS III at ARC 3.5 m, SP: SPIcam at ARC 3.5 m, LR: LRIS at Keck, NR: NIRC at Keck, UF: UFTI at UKIRT, SC: Suprime-Cam at Subaru, MS: MMT spectrograph, EF: EFOSC2 at ESO $3.6 \mathrm{~m}$.

References. (1) Hennawi et al. 2006; (2) Oguri et al. 2007b; (3) Oguri et al. 2005; (4) Walsh et al. 1979; (5) Inada et al. 2003b; (6) Oguri et al. 2004a; (7) Croom et al. 2004; (8) Pindor et al. 2006.
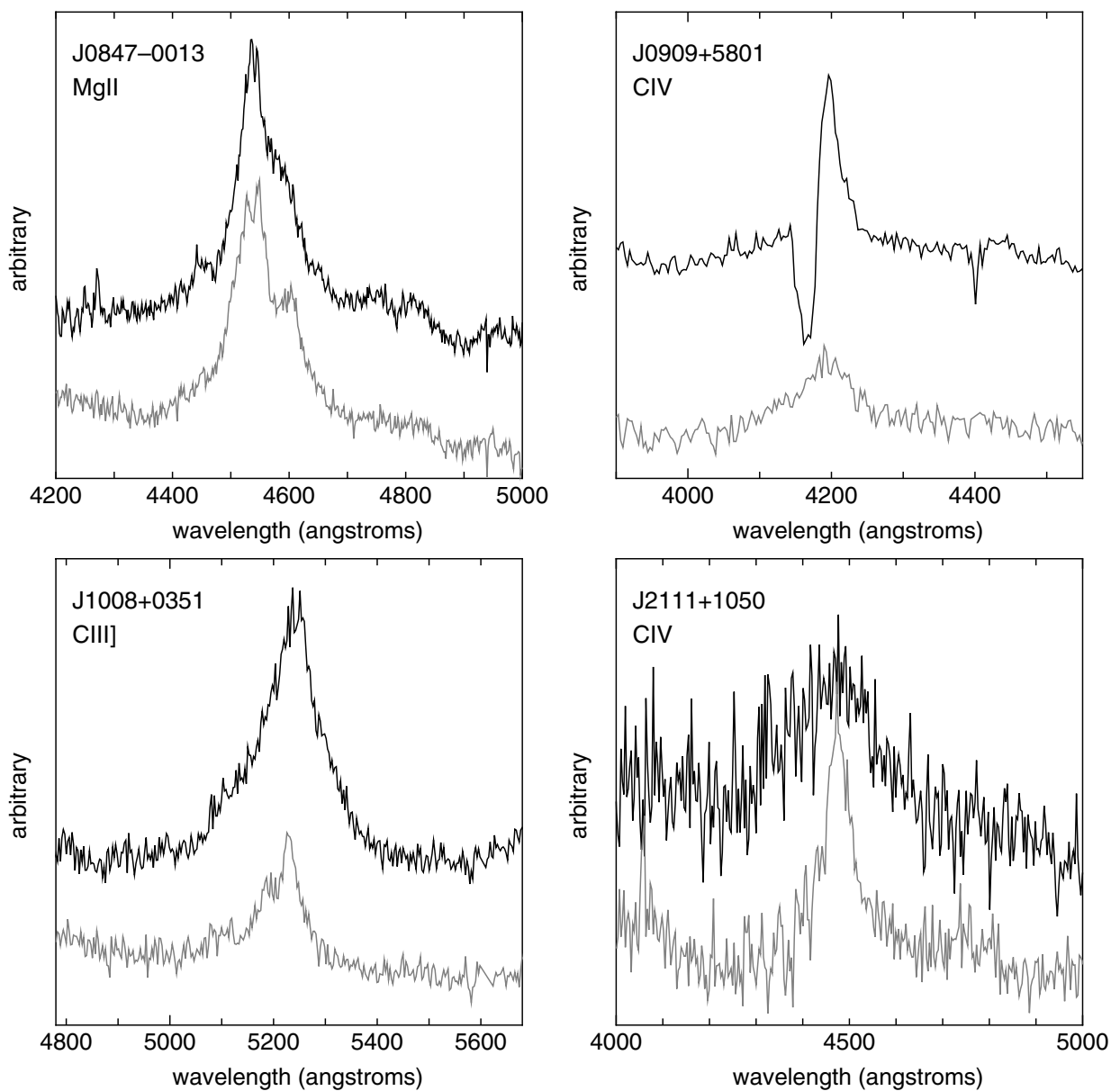

Figure 3. Upper left: the Mg II emission lines of the two quasar components of SDSS J0847-0013 taken at the Keck telescope (spectral resolution of $R \sim 1000$ ). The different shapes support the binary interpretation of this system. Upper right: The C IV emission lines of the two quasar components of SDSS J0909+5801 taken at the ARC $3.5 \mathrm{~m}$ telescope $(R \sim 500)$. The broad absorption line feature is seen only in the spectrum of the brighter component, which suggests that this is a binary quasar rather than a lens. Lower left: the C III emission lines of the two quasar components of SDSS J1008+0351 from the observation at the Subaru telescope $(R \sim 500)$. In addition to the different overall shapes, they show slightly different redshifts. Lower right: the C IV emission lines of the two quasar components of SDSS J2111+1050 observed at the ARC $3.5 \mathrm{~m}$ telescope $(R \sim 500)$. The different strengths of the emission lines, together with the absence of any lensing objects in the deep $I$-band image, support the binary interpretation.

\section{LENSED QUASARS}

After completing the observations, we have a statistical sample of 11 lensed quasars with image separations of $1^{\prime \prime}<$ $\theta<20^{\prime \prime}$ and $i$-band flux ratios (for two-image systems) of faint to bright lensed images larger than $10^{-0.5}$. Nine of them are newly discovered lensed quasars in the SQLS (e.g., Inada et al. 2005), and two of them, SDSS J0913+5259 (SBS 0909+523; 
Table 4

Lensed Quasars from the SDSS DR3: Statistical Sample

\begin{tabular}{lccrccc}
\hline \hline Object & $N_{\text {img }}$ & \multicolumn{1}{c}{$z^{\mathrm{a}}$} & $\theta_{\max }{ }^{\mathrm{b}}$ & $f_{i}$ or $f_{I}{ }^{\mathrm{c}}$ & Comment & Ref. \\
\hline SDSS J0246-0825 & 2 & 1.682 & 1.04 & 0.34 & SDSS lens & 1 \\
SDSS J0913+5259 & 2 & 1.377 & 1.14 & 0.70 & Known lens SBS 0909+523 & 2,3 \\
SDSS J0924+0219 & 4 & 1.524 & 1.78 & 0.43 & SDSS lens & 4 \\
SDSS J1001+5027 & 2 & 1.838 & 2.86 & 0.72 & SDSS lens & 5 \\
SDSS J1001+5553 & 2 & 1.405 & 6.17 & 0.94 & Known lens Q0957+561 & 3,6 \\
SDSS J1004+4112 & 5 & 1.732 & 14.62 & 0.23 & SDSS lens & 7,8 \\
SDSS J1021+4913 & 2 & 1.720 & 1.14 & 0.40 & SDSS lens & 9 \\
SDSS J1226-0006 & 2 & 1.121 & 1.24 & 0.45 & SDSS lens & 10 \\
SDSS J1332+0347 & 2 & 1.445 & 1.14 & 0.70 & SDSS lens & 11 \\
SDSS J1335+0118 & 2 & 1.570 & 1.56 & 0.37 & SDSS lens & 12 \\
SDSS J1524+4409 & 2 & 1.210 & 1.67 & 0.56 & SDSS lens & 13 \\
\hline
\end{tabular}

Notes.

a Redshifts from the follow-up observations.

${ }^{\mathrm{b}}$ Maximum image separations in units of arcsec.

${ }^{c}$ Flux ratios between the brightest lensed image and the farthest lensed image from the brightest image, in the $I$ - or $i$-band images.

References. (1) Inada et al. 2005; (2) Oscoz et al. 1997; (3) CASTLES webpage (C. S. Kochanek et al., http://cfa-www.harvard.edu/castles/.); (4) Inada et al. 2003a; (5) Oguri et al. 2005; (6) Walsh et al. 1979; (7) Inada et al. 2003b; (8) Oguri et al. 2004a; (9) Pindor et al. 2006; (10) Inada et al. 2007b; (11) Morokuma et al. 2007; (12) Oguri et al. (2004b); (13) Oguri et al. 2008b.

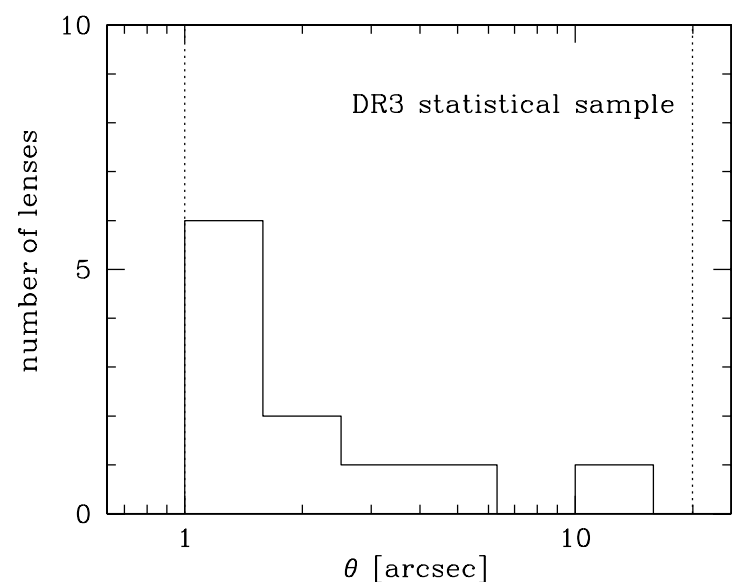

Figure 4. Image separation distribution of the SQLS DR3 statistical sample in bins of $\Delta \log \theta=0.2$. The statistical sample is constructed in the range $1^{\prime \prime}<\theta<20^{\prime \prime}$ as indicated by the dotted lines. The individual lenses are listed in Table 4.

Oscoz et al. 1997) and SDSS 1001+5553 (Q0957+561; Walsh et al. 1979), are previously known lensed quasars. The statistical sample is drawn from a subsample $(22,683$ quasars with $0.6<$ $z<2.2$ and $\left.i_{\text {cor }}>19.1\right)$ of the 46,420 DR3 quasars, and therefore some of lenses outside the SDSS DR3 (e.g., Inada et al. 2006a, 2006b) are not included. Table 4 summarizes the DR3 statistical lensed quasar sample, and Figure 4 shows a histogram of the maximum image separations of each system. The number of the lenses decreases from $\theta=1^{\prime \prime}$ as the image separation increases, consistent with previous observations (e.g., Browne et al. 2003). The statistical sample contains nine double lenses and two quadruple lenses, and ranges from $\theta=1$." 0 to $\theta=14$." 6 , covering both galaxy- and cluster-scale lenses.

There are two lensed quasars listed in Table 2 or 3 that are not part of our statistical sample in Table 4. SDSS J0832+0404 (Oguri et al. 2008b) is a color-selected SDSS lens but it is not included in the statistical sample because its $I$-band flux ratio is too extreme. The previously known lensed quasar SDSS J1633+3134 (FBQ 1633+3134; Morgan et al. 2001) is a morphologically-selected lens, but its image separation of $\theta=00^{\prime \prime} 66$ is too small to be included in the statistical sample. These two lenses are listed in Table 5, which contains additional DR3 lensed quasars outside the statistical sample.

We also applied our selection algorithms to the DR3 spectroscopic quasars outside the subsample of 22,683 quasars. For the higher redshift quasars $(z>2.2)$, we used the star_L criteria for the griz bands rather than for the ugri bands of the lower redshift lens selection. We found four SDSS lenses, SDSS J0903+5028 (Johnston et al. 2003), SDSS J1138+0314 (Burles et al. 2007), SDSS J1155+6346 (Pindor et al. 2004), and SDSS J1406+6126 (Inada et al. 2007a), and recovered two known lenses, SDSS J0145-0945 (Q0142-100; Reimers et al. 2002), and SDSS J0911+0550 (RX J0911+0551; Surdej et al. 1987). Note that SDSS J0903+5028 was first identified through its compound nature (quasar plus luminous red galaxy) of the SDSS spectrum (Johnston et al. 2003) and SDSS J1138+0314 was first identified in a WB $6.5 \mathrm{~m}$ snapshot survey of $\sim 1000$ SDSS quasars (Burles et al. 2007).

There are three lenses in the DR3 area which we did not recover as final follow-up candidate. In two cases, SDSS J0813+2545 (HS 0810+2554; Reimers et al. 2002) and SDSS J1650+4251 (Morgan et al. 2003), the objects are selected in the first stage of our morphological selection process and then eliminated by the GALFIT criteria which are designed to select lenses with $\theta>1^{\prime \prime}$ and $i$-band flux ratios of faint to bright lensed images larger than $10^{-0.5}$ (see Paper I). SDSS J0813+2545 (HS $0810+2554)$ has a small image separation $\left(<1^{\prime \prime}\right)$ and SDSS $\mathrm{J} 1650+4251$ has a small flux ratio $\left(<10^{-0.5}\right)$, that is why they did not pass the GALFIT fitting. The subarcsecond (0.68) radio lens (PMN J0134-0931; Winn et al. 2002; Gregg et al. 2002) was never flagged at any stage of our survey because of its small image separation. These three lenses would not be part of our statistical lens sample in any case. It does illustrate, however, that our survey is incomplete outside of our selection limits. 
Table 5

Additional Lensed Quasars in the SDSS DR3 Quasar Catalog

\begin{tabular}{lccccccc}
\hline \hline Object & $N_{\text {img }}$ & $z^{\mathrm{a}}$ & $\theta^{\mathrm{b}}$ & $f_{i}$ or $f_{I}^{\mathrm{c}}$ & Comment & Reject $^{\mathrm{d}}$ & Ref. $^{\mathrm{c}}$ \\
\hline SDSS J0134-0931 & 5 & 2.216 & 0.68 & 0.03 & Known lens PMN J0134-0931 & Too small $\theta$ & $1,2,3$ \\
SDSS J0145-0945 & 2 & 2.719 & 2.23 & 0.15 & Known lens Q0142-100 & $z>2.2, f_{I}<10^{-0.5}$ & 3,4 \\
SDSS J0813+2545 & 4 & 1.500 & 0.91 & 0.06 & Known lens HS 0810+2554 & By GALFIT fitting & 3,5 \\
SDSS J0832+0404 & 2 & 1.115 & 1.98 & 0.22 & SDSS lens & $f_{I}<10^{-0.5}$ & 6 \\
SDSS J0903+5028 & 2 & 3.584 & 2.80 & 0.46 & SDSS lens & $z>2.2$ & 7 \\
SDSS J0911+0550 & 4 & 2.800 & 3.26 & 0.41 & Known lens RX J0911+0551 & $z>2.2$ & 3,8 \\
SDSS J1138+0314 & 4 & 2.442 & 1.44 & 0.35 & SDSS lens & $z>2.2$ & 3,9 \\
SDSS J1155+6346 & 2 & 2.890 & 1.83 & 0.50 & SDSS lens & $z>2.2$ & 3,10 \\
SDSS J1406+6126 & 2 & 2.126 & 1.98 & 0.58 & SDSS lens & $i_{\text {cor }>19.1}$ & 11 \\
SDSS J1633+3134 & 2 & 1.511 & 0.66 & 0.30 & Known lens FBQ 1633+3134 & $\theta<1^{\prime \prime}, f_{I}<10^{-0.5}$ & 12 \\
SDSS J1650+4251 & 2 & 1.547 & 1.20 & 0.17 & SDSS lens & By GALFIT fitting & 13 \\
\hline
\end{tabular}

Notes. See text for the selection of each lensed quasar.

${ }^{a}$ Redshifts from follow-up observations.

${ }^{\mathrm{b}}$ Maximum image separations in units of arcsec.

${ }^{\mathrm{c}}$ Flux ratios between the brightest lensed image and the farthest lensed image from the brightest image, in the $I$ - or $i$-band images.

$\mathrm{d}$ The reason that each lens is excluded from the statistical sample.

References. (1) Winn et al. 2002; (2) Gregg et al. 2002; (3) CASTLES webpage (C. S. Kochanek et al., http://cfa-www.harvard.edu/castles/.); (4) Surdej et al. 1987; (5) Reimers et al. 2002; (6) Oguri et al. 2008b; (7) Johnston et al. 2003; (8) Bade et al. 1997; (9) Burles et al. 2007; (10) Pindor et al. 2004; (11) Inada et al. 2007a; (12) Morgan et al. 2001; (13) Morgan et al. 2003.

\section{SUMMARY AND DISCUSSIONS}

We have presented a complete sample of gravitationally lensed quasars which can be used for various statistical studies. The sample is based on the 46,420 spectroscopic quasars in the SDSS DR3. We focused on a subsample of 22,683 quasars with $0.6<z<2.2$ and Galactic extinction-corrected $i$-band PSF magnitudes brighter than 19.1. Lens candidates were identified using the algorithms described here and in paper I, and verified by extensive follow-up observations in the optical and nearIR. The resulting 11 lensed quasars constitute a statistical sample with image separation of $1^{\prime \prime}<\theta<20^{\prime \prime}$ and flux ratios (for two-image systems) of $f_{i}>10^{-0.5}$ that should have very high completeness based on our tests in Paper I. The DR3 spectroscopic quasar catalog contains an additional 11 lensed quasars that do not satisfy the criteria for our complete sample. Thus we identified a total of 22 lenses, 7 of which were discovered in earlier lens searches other than the SDSS.

The lens fraction in our statistical sample, $\sim 0.05 \%$, appears to be lower than in previous studies, but this is largely explained by our tight criterion on the image separations and flux ratios and the fact that our sample uses relatively low-redshift faint quasars. For example, the CLASS survey found a lens fraction of $\sim 0.14 \%$ (Browne et al. 2003), but $\sim 30 \%$ of the lenses have separations smaller than $1^{\prime \prime}$ and $\sim 50 \%$ of the double image lenses have flux ratios of faint to bright lensed images smaller than $10^{-0.5}$. The HST Snapshot Survey (Maoz et al. 1993) found that $\sim 1 \%$ of bright quasars were lensed, but the magnification bias for bright quasars is significantly larger than for our fainter quasar sample.

This is the first statistical lensed quasar sample that contains both galaxy-scale $\left(\theta \lesssim 3^{\prime \prime}\right)$ lenses and cluster-scale $(\theta \gtrsim$ $\left.10^{\prime \prime}\right)$ lenses. The maximum image separation in our DR3 statistical sample is 14".62 (SDSS J1004+4112), much larger than the maximum image separation in the CLASS, 4".56. The distribution of image separations across the wide mass range from galaxies to clusters will be valuable in studying the structure formation. We note that such distributions of splitting angles are obtained from surveys of lensed galaxies as well. For instance, Cabanac et al. (2007) presented 40 strongly lensed galaxy candidates, with separations ranging from $2^{\prime \prime}$ to $15^{\prime \prime}$. The simple point-like nature of source quasars, however, makes it much easier to quantify various selection effects than in surveys of lensed galaxies. In addition, our statistical sample has an advantage over lensed galaxies (or radio lenses) in wellunderstood redshift distribution of the source population. As an application of the statistical sample, we study cosmological constraints from the galaxy-scale lenses of the DR3 statistical lens sample in Paper III.

The DR3 spectroscopic quasar sample consists of less than half of the full SDSS data. Our lens sample will increase significantly in the future. Assembly of a complete lens sample from the DR5 quasar catalog (Schneider et al. 2007) is in progress.

N. I. acknowledges support from the Special Postdoctoral Researcher Program of RIKEN and the Japan Society for the Promotion of Science. This work was supported in part by Department of Energy contract DE-AC02-76SF00515. A portion of this work was performed under the auspices of the U.S. Department of Energy by Lawrence Livermore National Laboratory under contract DE-AC52-07NA27344. M. A. S. acknowledges support from NSF grant AST 03-07409. I. K. acknowledges supports from Ministry of Education, Culture, Sports, Science, and Technology, Grant-in-Aid for Encouragement of Young Scientists (No. 17740139), and Grant-in-Aid for Scientific Research on Priority Areas No. 467 "Probing the Dark Energy through an Extremely Wide \& Deep Survey with the Subaru Telescope." A. C. acknowledges the support of CONICYT, Chile, under grant FONDECYT 1051061.

Use of the UH $2.2 \mathrm{~m}$ telescope and the UKIRT $3.8 \mathrm{~m}$ telescope for the observations is supported by the National Astronomical Observatory of Japan (NAOJ). Based in part on observations obtained with the Apache Point Observatory $3.5 \mathrm{~m}$ telescope, which is owned and operated by the 
Astrophysical Research Consortium. Based in part on data collected at Subaru Telescope (some of data obtained from the Subaru Telescope Sciences Archive System (SMOKA)), which is operated by NAOJ. Some of the data presented herein were obtained at the W. M. Keck Observatory, which is operated as a scientific partnership among the California Institute of Technology, the University of California and the National Aeronautics and Space Administration. The Keck Observatory was made possible by the generous financial support of the W. M. Keck Foundation. The WIYN Observatory is a joint facility of the University of Wisconsin-Madison, Indiana University, Yale University, and the National Optical Astronomy Observatories. This work is also based in part on observations obtained with the MDM $2.4 \mathrm{~m}$ Hiltner telescope, which is owned and operated by a consortium consisting of Columbia University, Dartmouth College, the University of Michigan, the Ohio State University and Ohio University. The WB $6.5 \mathrm{~m}$ telescope is the first telescope of the Magellan Project; a collaboration between the Observatories of the Carnegie Institution of Washington, University of Arizona, Harvard University, University of Michigan, and Massachusetts Institute of Technology to construct two $6.5 \mathrm{~m}$ optical telescopes in the southern hemisphere. Based in part on observations made with the NASA/ESA HST, obtained at the Space Telescope Science Institute, which is operated by the Association of Universities for Research in Astronomy, Inc., under NASA contract NAS 5-26555. These observations are associated with $H S T$ program GO-9744. Based in part on observations made with telescopes (ESO $3.6 \mathrm{~m}$ and NTT) at the European Southern Observatories La Silla in Chile. Some observations reported here were obtained at the MMT Observatory, a joint facility of the University of Arizona and the Smithsonian Institution.

Funding for the SDSS and SDSS-II has been provided by the Alfred P. Sloan Foundation, the Participating Institutions, the National Science Foundation, the U.S. Department of Energy, the National Aeronautics and Space Administration, the Japanese Monbukagakusho, the Max Planck Society, and the Higher Education Funding Council for England. The SDSS Web site is http://www.sdss.org/.

The SDSS is managed by the Astrophysical Research Consortium for the Participating Institutions. The Participating Institutions are the American Museum of Natural History, Astrophysical Institute Potsdam, University of Basel, Cambridge University, Case Western Reserve University, University of Chicago, Drexel University, Fermilab, the Institute for Advanced Study, the Japan Participation Group, Johns Hopkins University, the Joint Institute for Nuclear Astrophysics, the Kavli Institute for Particle Astrophysics and Cosmology, the Korean Scientist Group, the Chinese Academy of Sciences (LAMOST), Los Alamos National Laboratory, the Max Planck Institute for Astronomy (MPIA), the Max Planck Institute for Astrophysics (MPA), New Mexico State University, Ohio State University, University of Pittsburgh, University of Portsmouth, Princeton University, the United States Naval Observatory, and the University of Washington.

\section{APPENDIX}

The redshift and $i_{\text {cor }}$ distributions of our quasar subsample are presented in their entirety in the online journal. A portion is shown here (Table 6) for guidance regarding its form and content.
Table 6

Redshifts and Galactic Extinction Corrected $i$-band Magnitudes of the 22,683 Quasars

\begin{tabular}{lcc}
\hline \hline Name & $z$ & $i_{\text {cor }}$ \\
\hline SDSSJ000009.26+151754.5 & 1.19860 & 19.07468 \\
SDSSJ000009.42-102751.9 & 1.84420 & 18.71486 \\
SDSSJ000013.14+141034.6 & 0.94910 & 19.02986 \\
SDSSJ000017.38-085123.7 & 1.24990 & 18.19743 \\
SDSSJ000024.02+152005.4 & 0.98850 & 18.98949
\end{tabular}

(This table is available in its entirety in a machinereadable form in the online journal. A portion is shown here for guidance regarding its form and content)

\section{REFERENCES}

Abazajian, K., et al. 2003, AJ, 126, 2081

Abazajian, K., et al. 2004, AJ, 128, 502

Abazajian, K., et al. 2005, AJ, 129, 1755

Adelman-McCarthy, J. K., et al. 2006, ApJS, 162, 38

Adelman-McCarthy, J. K., et al. 2007, ApJS, 172, 634

Bade, N., Siebert, J., Lopez, S., Voges, W., \& Reimers, D. 1997, A\&A, 317, L13

Becker, R. H., White, R. L., \& Helfand, D. J. 1995, ApJ, 450, 559

Browne, I. W. A., et al. 2003, MNRAS, 341, 13

Burles, S., et al. 2007, in preparation

Cabanac, R. A., et al. 2007, A\&A, 461, 813

Chae, K.-H., et al. 2002, Phys. Rev. Lett., 89, 151301

Chae, K.-H., Mao, S., \& Kang, X. 2006, MNRAS, 373, 1369

Chen, D.-M. 2004, A\&A, 418, 387

Croom, S. M., Smith, R. J., Boyle, B. J., Shanks, T., Miller, L., Outram, P. J., \& Loaring, N. S. 2004, MNRAS, 349, 1397

Falco, E. E., et al. 1999, ApJ, 523, 617

Fukugita, M., Shimasaku, K., \& Ichikawa, T. 1995, PASP, 107, 945

Fukugita, M., Ichikawa, T., Gunn, J. E., Doi, M., Shimasaku, K., \& Schneider, D. P. 1996, AJ, 111, 1748

Gregg, M. D., Lacy, M., White, R. L., Glikman, E., Helfand, D., Becker, R. H., \& Brotherton, M. S. 2002, ApJ, 564, 133

Gregg, M. D., et al. 2007, in preparation

Gunn, J. E., et al. 1998, AJ, 116, 3040

Gunn, J. E., et al. 2006, AJ, 131, 2332

Hennawi, J. F., et al. 2006, AJ, 131, 1

Hogg, D. W., Finkbeiner, D. P., Schlegel, D. J., \& Gunn, J. E. 2001, AJ, 122,2129

Inada, N., et al. 2003a, AJ, 126, 666

Inada, N., et al. 2003b, Nature, 426, 810

Inada, N., et al. 2005, AJ, 130, 1967

Inada, N., et al. 2006a, AJ, 131, 1934

Inada, N., et al. 2006b, ApJ, 653, L97

Inada, N., et al. 2007a, AJ, 133, 206

Inada, N., et al. 2007b, AJ, in preparation

Ivezić, Ž., et al. 2004, AN, 325, 583

Johnston, D. E., et al. 2003, AJ, 126, 2281

Kochanek, C. S., Falco, E. E., \& Muñoz, J. A. 1999, ApJ, 510, 590

Kochanek, C. S., \& White, M. 2001, ApJ, 559, 531

Kochanek, C. S., Schneider, P., \& Wambsganss, J. 2006, in Proc. 33rd Saas-Fee Advanced Course, Part 2 of Gravitational Lensing: Strong, Weak \& Micro, ed. G. Meylan, P. Jetzer, \& P. North (Berlin: Springer), 91

Lupton, R. 2005, AJ, submitted

Lupton, R., Gunn, J. E., Ivezić, Z., Knapp, G. R., Kent, S., \& Yasuda, N. 2001, in ASP Conf. Ser. 238, Astronomical Data Analysis Software and Systems X, ed. F. R. Harnden, Jr., F. A. Primini, \& H. E. Payne (San Francisco, CA: ASP), 269

Maoz, D., et al. 1993, ApJ, 409, 28

Mitchell, J. L., Keeton, C. R., Frieman, J. A., \& Sheth, R. K. 2005, ApJ, 622, 81 Morgan, N. D., Becker, R. H., Gregg, M. D., Schechter, P. L., \& White, R. L. 2001, AJ, 121, 611

Morgan, N. D., Snyder, J. A., \& Reens, L. H. 2003, AJ, 126, 2145

Morokuma, T., et al. 2007, AJ, 133, 214 
Myers, S. T., et al. 2003, MNRAS, 341, 1

Muñoz, J. A., Falco, E. E., Kochanek, C. S., McLeod, B. A., \& Mediavilla, E. 2003, ApJ, 605, 614

Oguri, M. 2006, MNRAS, 367, 1241

Oguri, M., et al. 2004a, ApJ, 605, 78

Oguri, M., et al. 2004b, PASJ, 56, 399

Oguri, M., et al. 2005, ApJ, 622, 106

Oguri, M., et al. 2006, AJ, 132, 999 (Paper I)

Oguri, M., et al. 2008a, AJ, 135, 512 (Paper III)

Oguri, M., et al. 2008b, AJ, 135, 520

Oscoz, A., Serra-Ricart, M., Mediavilla, E., Buitrago, J., \& Goicoechea, L. J. 1997, ApJ, 491, L7

Padmanabhan, N., et al. 2008, in press (arXiv astro/ph/0703454)

Peng, C. Y., Ho, L. C., Impey, C. D., \& Rix, H.-W. 2002, AJ, 124, 266

Phillips, P. M., et al. 2001, MNRAS, 328, 1001

Pier, J. R., Munn, J. A., Hindsley, R. B., Hennessy, G. S., Kent, S. M., Lupton, R. H., \& Ivezić, Ź. 2003, AJ, 125, 1559

Pindor, B., et al. 2004, AJ, 127, 1318

Pindor, B., et al. 2006, AJ, 131, 41
Reimers, D., Hagen, H.-J., Baade, R., Lopez, S., \& Tytler, D. 2002, A\&A, 382, L26

Richards, G. T., et al. 2002, AJ, 123, 2945

Richards, G. T., et al. 2006, AJ, 131, 2766

Rusin, D. 2002, ApJ, 572, 705

Rusin, D., \& Tegmark, M. 2001, ApJ, 553, 709

Schlegel, D. J., Finkbeiner, D. P., \& Davis, M. 1998, ApJ, 500, 525

Schneider, D. P., et al. 2005, AJ, 130, 367

Schneider, D. P., et al. 2007, AJ, 134, 102

Smith, J. A., et al. 2002, AJ, 123, 2121

Stoughton, C., et al. 2002, AJ, 123, 485

Surdej, J., Swings, J.-P., Magain, P., Courvoisier, T. J.-L., \& Borgeest, U. 1987, Nature, 329, 695

Tucker, D. L., et al. 2006, AN, 327, 821

Vanden Berk, D. E., et al. 2005, AJ, 129, 2047

Walsh, D., Carswell, R. F., \& Weymann, R. J. 1979, Nature, 279, 381

Winn, J. N., Lovell, J. E. J., Chen, H.-W., Fletcher, A. B., Hewitt, J. N., Patnaik, A. R., \& Schechter, P. L. 2002, ApJ, 564, 143

York, D. G., et al. 2000, AJ, 120, 1579 